Research Article

\title{
Wavelet Transform-Based Damage Identification in Bladed Disks and Rotating Blades
}

\author{
P. Rajendran, ${ }^{1,2}$ N. Jamia, ${ }^{3}$ S. El-Borgi $\left(\mathbb{D},{ }^{1}\right.$ and M. I. Friswell ${ }^{3}$ \\ ${ }^{1}$ Mechanical Engineering Program, Texas A\&M University at Qatar, Engineering Building, P.O. Box 23874, Education City, \\ Doha, Qatar \\ ${ }^{2}$ School of Mechanical Engineering, SASTRA Deemed University, Thanjavur 613401, Tamil Nadu, India \\ ${ }^{3}$ Swansea University, Bay Campus, Fabian Way, Swansea SA18EN, UK \\ Correspondence should be addressed to S. El-Borgi; sami.el_borgi@qatar.tamu.edu
}

Received 8 June 2018; Revised 31 August 2018; Accepted 19 September 2018; Published 17 October 2018

Academic Editor: Marcello Vanali

Copyright (c) 2018 P. Rajendran et al. This is an open access article distributed under the Creative Commons Attribution License, which permits unrestricted use, distribution, and reproduction in any medium, provided the original work is properly cited.

\begin{abstract}
Blade vibration and blade clearance are effective diagnostic features for the identification of blade damage in rotating machines. Blade tip-timing (BTT) is a noncontact method that is often used to monitor the vibration and clearance of blades in a rotating machinery. Standard signal processing of BTT measurements give one blade response sample per revolution of the machine which is often insufficient for the diagnosis of damage. This paper uses the raw data signals from the sensors directly and employs a wavelet energy-based mistuning index (WEBMI) to predict the presence and locations of damage in rotating blades. The Lipschitz exponent is derived from the wavelet packet coefficients and used to estimate the severity of the damage. In this study, experiments were conducted to obtain BTT measurements on rotating blades at $100 \mathrm{rpm}$ using three different sensors: an active eddy current sensor, a passive eddy current sensor, and an optical sensor. In addition, hammer excitation experiments were conducted for various added mass (damage) cases to compute the damage severity for a bladed disk. To simulate the damage experimentally in the bladed disk and rotating blades, masses were added to the blades to alter their dynamics and mimic the damage. The results indicate that the WEBMI can detect the presence and location of damage in rotating blades using measurements from common BTT sensors. To check the robustness of the proposed damage severity index, the experimental results were compared with numerical simulation for the bladed disk and showed good agreement.
\end{abstract}

\section{Introduction}

High cycle fatigue (HCF) causes damage to the blades during the operation of a turbomachine and if unnoticed may lead to catastrophic failures of the whole machine. As a result, there is an increase in maintenance cost and reduction in lead time. Therefore, an early assessment of the damage in rotating blades for condition-based maintenance improves the efficiency and increases the life of the turbomachine. Over the past few decades, many researchers have proposed noncontact measurement and data processing techniques to monitor the vibration and clearance of blades in rotating machines due to the nonintrusive and easy installation of sensors which allows prompt identification of potential damage. The blade tip-timing (BTT) method is a well-known noncontact method which measures the blade vibration and blade tip-clearance during the engine operation and detects the different types of blade failures [1]. This concept relies on a number of probes fixed to the casing of the machine to detect the blade tip as it passes in front of the probes. The effect of mistuned blades on the synchronous vibration of rotating blades has been identified by BTT data analysis [2] The blade vibration was monitored, and blade cracks were detected during machine operation [3]. The abnormalities found in the vibration characteristics of the BTT data measured during engine tests were used to identify blade damage [4]. BTT measurements were employed for identifying the dynamic behavior and detecting a small mistuning pattern of rotating bladed disks [5]. Along with blade vibration data, the blade tip-clearance (BTC) measurement, which is the gap between the blade tip and the casing of a rotating machine, is an additional diagnostic parameter 
that has been used to identify damage. These measurements can also be obtained by the blade tip sensors.

Several blade vibration monitoring technologies have been summarized which distinguish between the effect of the cracks and any other source of damage based on the blade length measurements [1]. The tip timing data of a rotor blade were measured in various engine trials to evaluate the ability of these sensors to detect dynamic foreign object damage events [6]. A contactless diagnostic method was described to identify damage in steam turbine blades based on the axial blade lengthening parameter [7]. However, it is challenging to predict the anomalies in the rotating blades using only blade lengthening and amplitude parameters. Hence, data-driven detection techniques were employed to analyze BTC data acquired from the rotor disk to test the feasibility of the damage detection methodology [8].

In addition to the BTC data, the modal parameters of blades were utilized to detect damage in the rotating blades. The experimental studies were performed on the assembled test rig of three spinning test blades, and the location and length of a crack were estimated based on the modal parameters [9]. The blade natural frequencies and the blade tip position were obtained from the BTT method to detect the blade crack in an aero engine [10]. Chatterjee and Kotambkar [11] investigated the mistuning induced by lacing wire damage in blade packets using modal characteristics. Xu et al. [12] studied the effect of cracks on the vibration characteristics of mistuned rotating blades based on natural frequency, vibration amplitude, and vibration localization parameters. Nevertheless, the modal parameters are less sensitive to the low damage levels, and the natural frequencies and mode shapes are difficult to measure due to the subsampling frequency of the BTT signals, which depends on the rotating speed. Thus, the signal processing of BTT measurements is necessary to reconstruct the signal to solve the aliasing effect before applying into damage detection algorithms. Salhi et al. [13] proposed a BTT signal reconstruction method based on the Shannon theorem. However, in practice, BTT signals are not really band limited, and so aliasing effects still exist in the reconstructed signals.

The low sample rate of BTT signals occurs because only one blade response sample per revolution is extracted from the processing of the raw sensor signal. A sparse representation (SR) model was built for multimode blade vibration monitoring $[14,15]$. However, the sensor output is often measured at a high sample rate and will contain a combination of the blade vibration and the rotation of the machine. Extracting suitable features from these signals to detect damage requires advanced signal processing tools, such as the wavelet transform.

The wavelet packet transform (WPT) is a rich signalprocessing tool which performs a complete decomposition, including both low-frequency (approximate) and highfrequency (detailed) components. The WPT can also handle the subsampled signals and overcome the aliasing effects in the reconstructed signals. Zhongsheng [16] proposed a nonaliasing reconstruction algorithm of subsampled blade tip-timing signals based on the Shannon theorem and WPT. The WPT was applied to subsampled rotational mode shapes to detect the location and size of added mass in beam and plate structures by numerical simulation $[17,18]$. Jamia et al. [19] employed the WPT analysis to identify the presence, location, and severity of mistuning in a bladed disk by conducting a series of impact hammer tests. They mainly addressed the presence and location of damage but they did not justify the relationship between the damage severity and wavelet coefficients.

For damage severity estimation, Montanari et al. [20] introduced continuous wavelet transform- (CWT-) based crack index to detect and estimate the crack location and size of different beams using first three mode shapes. Hong et al. [21] introduced the Lipschitz exponent which can be used as a damage extent indicator in a beam using the CWT. Douka et al. [22] and Loutridis et al. [23] introduced an intensity factor that estimates the size of the crack from the coefficients of the CWT. The Lipschitz (Hoelder) exponent $(\alpha)$ and intensity factor $(K)$ were derived from the CWT coefficients to quantify the relationship between the damage and the change in the wavelet coefficients derived from modal and elemental strain energy data [24]. Chen et al. [25] employed a Lipschitz exponent to quantify the signal singularity of the acceleration responses of a fivestorey building model using the CWT analysis. For practical applications, a fast numerical algorithm is recommended; in order to obtain the discrete data points, one should sample the continuous wavelet algorithm on a dyadic grid, because it was proved that the wavelet transform on a dyadic grid is complete and stable [26]. Błaszczuk and Pozorski [27] discussed the application of the discrete wavelet transform (DWT) and the Lipschitz exponent to estimate the function differentiability of the beam structural response.

In summary, based on the above literature review, the following points can be concluded: (a) blade vibration and blade tip-clearance are effective diagnostic features for detecting blade damage in a rotating machine, (b) direct BTT signals have difficulty in interpreting damage in rotating blades, (c) modal parameters are insensitive to identify mistuning or damage in rotating blades, (d) the signal processing of BTT measurements is required before applying the WPT analysis, (e) the WPT analysis is a rich signal-processing tool which can even handle BTTreconstructed signals to detect anomalous features, and (f) the Lipschitz exponent has been employed to quantify damage. In light of this, the novelty of this study is to apply the raw signals from BTT sensors into a WPT analysis to detect the presence and location of damage in rotating blades and estimate the Lipschitz exponents from the wavelet packet coefficients to quantify the severity of damage in the bladed disk and rotating blades. An initial study is also reported that demonstrates the effectiveness of the Lipschitz exponent to quantify damage in stationary bladed disks using impact tests with the response measured by accelerometers.

The paper is organized as follows. Section 2 describes the formulation of the proposed WEBMI and the damage 
severity index. The performance of impact hammer tests to estimate the damage severity of bladed disks is briefly explained in Section 3. Details of the BTT experiments and measurement results are presented and discussed in Section 4. Finally, a summary and concluding remarks of this study are provided in Section 5.

\section{Wavelet Energy-Based Mistuning Index}

In this study, the wavelet energy-based mistuning index (WEBMI) is employed to detect the presence and location of damage in the rotating blades using measurements from the BTT sensors. For the sake of understanding, the WEBMI is briefly discussed, along with the threshold based on the statistical confidence. The description of the Lipschitz exponent is then given, followed by the definition of the proposed severity index.

2.1. Introduction of the WPT. The wavelet packet $\psi_{j, k}^{i}$ is a function where $i, j$, and $k$ are the frequency modulation, scale (decomposition level), and translation parameters, respectively. Thus

$$
\psi_{j, k}^{i}=2^{j / 2} \psi^{i}\left(2^{j} t-k\right),
$$

where $\psi^{i}$ indicates the frequency modulation of the wavelet function. After the $j$ th level of decomposition, the original signal $f(t)$ can be expressed as

$$
f(t)=2^{j} \sum_{i=1} f_{j}^{i}(t)
$$

The wavelet packet component signal $f_{j}^{i}(t)$ can be represented by a linear combination of the wavelet packet functions $\psi_{j, k}^{i}(t)$ as

$$
f_{j}^{i}(t)=N_{s} \sum_{k=0} C_{j, k}^{i}(t) \cdot \psi_{j, k}^{i}(t)
$$

where $N_{s}$ denotes the number of samples for four revolutions, and the wavelet packet coefficient $C_{j, k}^{i}$ can be obtained from

$$
\begin{aligned}
& C_{j, k}^{i}=\int_{0}^{T} f(t) \psi_{j, k}^{i}(t) d t, \\
& C_{j, k}^{i}=A_{j, k}^{i}, \quad \text { if } i=1,3,5 \ldots, \\
& C_{j, k}^{i}=D_{j, k}^{i}, \quad \text { if } i=2,4,6 \ldots,
\end{aligned}
$$

where $A_{j, k}^{i}$ and $D_{j, k}^{i}$ are the approximate and detail coefficients, respectively. The wavelet packet functions are assumed to be orthogonal, that is

$$
\int_{0}^{T} \psi_{j, k}^{r}(t) \psi_{j, k}^{q}(t) d t=0, \quad \text { if } r \neq q .
$$

Thus, the wavelet component energy at the $j$ th level for the $i$ th modal component is defined as the energy stored in the component signal $f_{j}^{i}(t)$ and is given by

$$
E_{j}^{i}=\int_{0}^{T} f_{j}^{i}(t)^{2} d t .
$$

The component signal $f_{j}^{i}(t)$ is extracted from the $j$ th level but is translated in the time domain such that $0<t<T$. The estimated component energy $E_{j}^{i}$ is the energy stored in different frequency bands at the $j$ th scale. The rate of change of the signal wavelet component energy $\Delta E$ at the $j$ th level and the $i$ th component can be expressed as

$$
\Delta E_{j}^{i}=\frac{\left|\left(E_{j}^{i}\right)_{d}-\left(E_{j}^{i}\right)_{u}\right|}{\left(E_{j}^{i}\right)_{u}},
$$

where the subscripts $d$ and $u$ denote the damaged and undamaged cases, respectively. The rate of change in energy, $\triangle E$, defines the WEBMI.

2.2. Threshold. To increase the robustness of prediction of the damage location, a threshold is established using the statistical properties and the one-sided confidence limit of the damage indices from successive measurements. The mean and standard deviation of the estimated WEBMI values are given by $\mu_{W}$ and $\sigma_{W}$, respectively. Thus, the onesided upper confidence limit for the WEBMI is given by the following equation [28]:

$$
U L_{W}=\mu_{W}+Z_{\beta}\left(\frac{\sigma_{W}}{\sqrt{N}}\right)
$$

where $N$ is the total number of WEBMI values that can be obtained after completing the wavelet packet decomposition at a given level. $Z_{\beta}$ is the value of the standard normal distribution with zero mean and unit variance that gives a cumulative probability of $100(1-\beta) \%$. This upper limit can be defined as a threshold value, and any index that exceeds the threshold can indicate the exact location of damage. The brief damage detection methodology using the WPT analysis is provided by the flowchart shown in Figure 1 .

2.3. The Lipschitz Exponent. A function $f(t)$ has a Lipschitz exponent $\alpha \geq 0$ at the point $t=v$ if there exists a constant $D$ and a polynomial $p_{v}(t)$ of degree $M$ (where $M$ is the largest integer satisfying $M \leq \alpha)$ such that [21]

$$
f(t)=p_{v}(t)+\varepsilon_{v}(t),
$$

where

$$
\left|\varepsilon_{v}(t)\right| \leq D|t-v| \alpha
$$

The Lipschitz exponent $\alpha$ measures the differentiability of the function $f(t)$; the function is not differentiable at $t=v$ if $0<\alpha<1$. All of the discontinuities in the function $f(t)$ at the point $t=v$ are contained in the function $\varepsilon_{v}(t)$.

When the Lipschitz exponent is applied to the wavelet transform, then determining the moments of the mother wavelet that vanish is important. A function $\psi(t)$ is said to have $n$ vanishing moments if it satisfies the following condition:

$$
\int_{-\infty}^{+\infty} t^{k} \psi(t) d t=0, \quad \text { for } 0 \leq k \leq n
$$




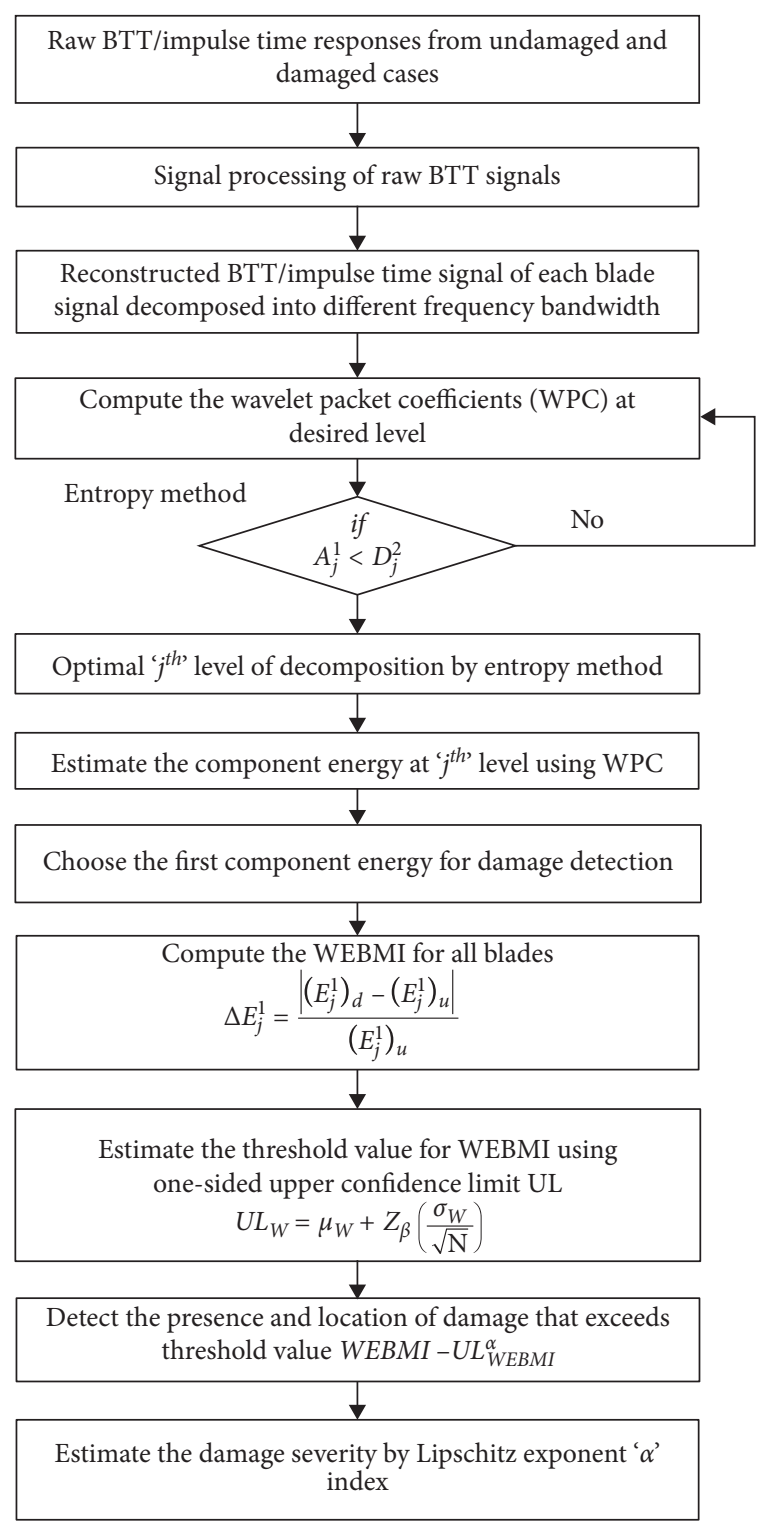

Figure 1: Damage detection methodology.

Suppose that the number of vanishing moments of the wavelet function is greater than the Lipschitz exponent, i.e., $n>\alpha$. Then, the wavelet transform retains only the singular part of the function $f(t)$ because

$$
W p_{v}(t)=0
$$

and hence

$$
W f(t)=W \varepsilon_{v}(t),
$$

where $W$ defines the wavelet transform.

The maximum wavelet packet coefficients reduce as the level of decomposition, $j$, decreases. For isolated singularities, the wavelet maximum is bounded by an exponential decay law, with an exponent equal to the Lipschitz exponent $\alpha$. Błaszczuk and Pozorski [27] proposed an inequality in the form

$$
|W f(t)| \leq D \times 2^{-j(\alpha+(1 / 2))}
$$

or equivalently

$$
\log _{2}|W f(t)| \leq-j\left(\alpha+\frac{1}{2}\right)+\log _{2} D
$$

Thus, a plot of $|W f(t)|$ against $j$ gives a straight line with slope $-(\alpha+1 / 2)$, and this slope can be estimated from two values of $j$.

2.4. The Damage Index. The key difference in the current analysis is to replace the wavelet packet coefficient, $W f(t)$, by the absolute difference between the WEBMI and the upper control limit, $|W E B M I-U L|$. The energy at any level of decomposition depends directly on the wavelet coefficients, and thus if damage causes a discontinuity in the wavelet transform, then it will also provide a discontinuity in the WEBMI. The estimate of the Lipschitz exponent in terms of the WEBMI is given by

$$
\begin{aligned}
\alpha= & \frac{-\log _{2}\left|(W E B M I-U L)_{j=j_{2}}\right|+\log _{2}\left|(W E B M I-U L)_{j=j_{1}}\right|}{j_{2}-j_{1}} \\
& -\frac{1}{2},
\end{aligned}
$$

where $j_{1}$ and $j_{2}$ are the decomposition levels chosen for the estimation.

\section{A Bladed Disk Example}

This study is an extension of our previous work that identified mistuning in a bladed disk using the WPT methodology [19]. To quantify the damage, the proposed damage severity index is now demonstrated on a bladed disk example. The simulation consists of a disk with 20 blades and two degrees of freedom per blade as shown in Figure 2, where the damage is simulated by a stiffness reduction of one blade. The experimental example consists of a disk with 12 blades, where the "damage" is implemented by adding mass to one of the blades as shown in Figure 3. The equivalence of the damage in the simulation and experiment is then determined by considering the change in the first natural frequency of the blade.

3.1. Numerical Simulation. The numerical simulation was performed on a reduced-order two degrees of freedom per blade model of the bladed disk developed by Salhi et al. [29] and used by Jamia et al. [19] to demonstrate the WEBMI. The bladed disk was composed of 20 blades. Figure 2 shows a schematic of the two DOF models, where only three sectors of the bladed disk are shown as a mass-spring system. An impulse was applied to one blade, and the corresponding time response was obtained from each blade tip. The output only response signal was used in the WPT analysis to obtain the WEBMI. The Lipschitz exponent was derived from the WPT analysis to estimate the damage severity in the bladed disk. 


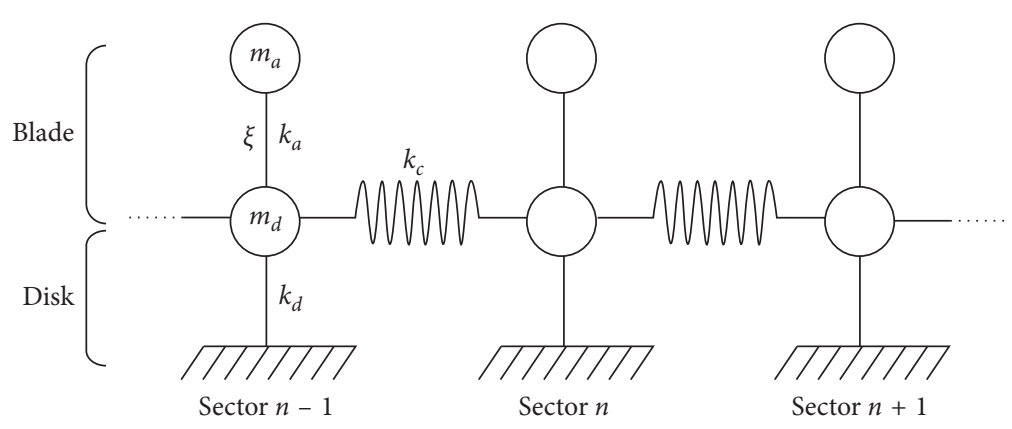

Figure 2: Two degrees of freedom per sector model.

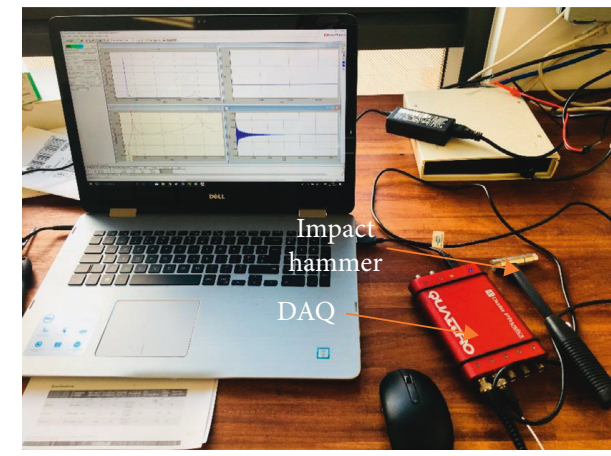

(a)

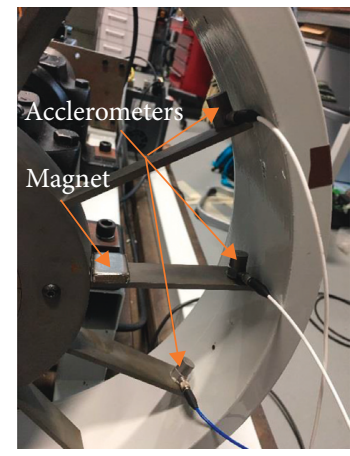

(b)

FIGURE 3: (a) Impact hammer test setup; (b) blade with added magnet mass.

The model used has two DOFs per sector, representing the blade and the disk sector. Each disk sector is coupled with the neighboring sectors by the stiffness $k_{c}$. The stiffness of an individual blade is represented by $k_{a}$. It is assumed that each blade in the assembly has one significant mode which is typically the first bending mode. No centrifugal stiffening or rotational dynamics effects are considered explicitly in this model, although centrifugal stiffening could be included by increasing the blade stiffness $k_{a}$. A proportional damping model is assumed. A free vibration response of each blade and disk sector is generated.

The equation of motion of the bladed disk model in free vibration is defined as

$$
\mathbf{M} \ddot{\mathbf{q}}(t)+\mathbf{C} \dot{q}(t)+\mathbf{K q}(t)=0,
$$

where $\mathbf{q}(t)$ is the vector of displacements corresponding to $(2 \times 20)$ DOF. The displacement of the $n$-th sector is given by

$$
\mathbf{q}^{n}(t)=\left[\begin{array}{l}
q_{1}^{n}(t) \\
q_{2}^{n}(t)
\end{array}\right]
$$

where the subscripts 1 and 2 represent the blade and the disk sector, respectively.

$\mathbf{M}, \mathbf{C}$, and $\mathbf{K}$ represent the structural mass, damping, and stiffness matrices of the dimension $N \times N$ with $N=2 n_{b}=40$, where $n_{b}$ is the number of blades. The mass matrix is given by

$$
\mathbf{M}=\left[\begin{array}{ccccc}
\mathbf{M}_{\mathbf{s}} & 0 & 0 & . & 0 \\
0 & \mathbf{M}_{\mathbf{s}} & 0 & . & 0 \\
0 & 0 & \mathbf{M}_{\mathbf{s}} & . & 0 \\
. & . & . & . & . \\
0 & 0 & . & . & \mathbf{M}_{\mathbf{s}}
\end{array}\right] \text { with } \quad \mathbf{M}_{\mathbf{s}}=\left[\begin{array}{cc}
m_{a} & 0 \\
0 & m_{d}
\end{array}\right]
$$

where $m_{a}$ and $m_{d}$ are the modal masses of the blade and the corresponding disk sector, respectively. The stiffness matrix $\mathbf{K}$ is given by

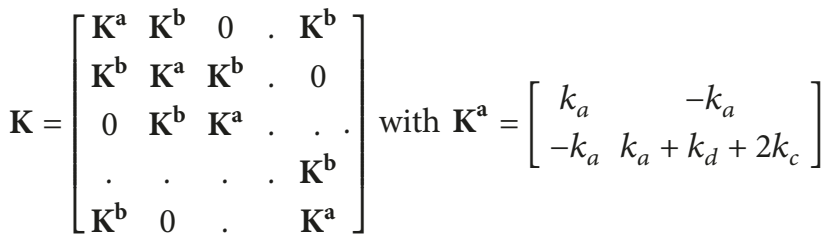

$$
\begin{aligned}
& \text { and } \mathbf{K}^{\mathbf{b}}=\left[\begin{array}{cc}
0 & 0 \\
0 & -k_{c}
\end{array}\right] \text {. }
\end{aligned}
$$

The proportional damping matrix is given by

$$
\mathbf{C}=\alpha \mathbf{M}+\beta \mathbf{K},
$$

where the proportionality factors $\alpha$ and $\beta$ are 1.0823 and $7.6536 \times 10^{-5}$, respectively. The damping ratios for all 
modes are approximately equal to 0.01 . A bladed disk is simulated with the parameters given in Table 1.

To examine the relationship between the Lipschitz exponent, $\alpha$, and the damage severity in the bladed disk, a numerical analysis was performed for different stiffness reductions that varied from $1 \%$ to $9 \%$. In this study, the wavelet function $d b 6$ was chosen which has three vanishing moments, and the first wavelet packet energy was considered in the damage identification methodology, as indicated in [19]. Then, the Lipschitz exponent $\alpha$ was computed with the input of two different levels $(j=2$ and $j=6$ ) of coefficients using Equation (16). The minimum level of $j=2$ was required to detect the damage and $j=6$ was chosen to satisfy the vanishing moments condition for the $d b 6$ wavelet. Higher-order wavelet functions were investigated but, in this case, the $d b 6$ wavelet is optimal to estimate the singularity of the function.

Figure 4 shows that the Lipschitz exponent $\alpha$ decreases exponentially as the stiffness reduction increases, which means the differentiability of the function decreases as the damage severity increases. The Lipschitz exponent $\alpha$ has an ability to predict the differentiability of the function for a stiffness change as small as $1 \%$. The Lipschitz exponent obeys the vanishing moments condition which means the chosen " $d b 6$ " wavelet function was suitable for this study. The minimum stiffness changes due to damage were $1 \%$ in this case; smaller stiffness changes will generally require higher order wavelet functions.

3.2. Experimental Validation. To check the robustness of the proposed damage severity index, an impact hammer test was conducted at Swansea University. To perform this experiment, a 4-channel data acquisition (DAQ) system, three accelerometers, an impact hammer, and data acquisition software were used (see Figure 3(a)), and the experimental procedure was briefly explained in [19]. In this study, the added mass was varied from $11 \mathrm{~g}$ to $99 \mathrm{~g}$, with an increment of $11 \mathrm{~g}$, on the middle blade to estimate the added mass (damage) severity as shown in Figure 3(b). The mass was added using rectangular magnets of mass $33 \mathrm{~g}$ shown in Figure 3(b), and circular magnets of mass $11 \mathrm{~g}$, mounted on the beam at centre position approximately $15 \mathrm{~mm}$ from the blade root. Table 2 shows the combination and location of the magnets to realise the different mass values. The experimental Lipschitz exponent, $\alpha$, was estimated and is shown in Figure 4 as a function of the mass added. The results clearly show that the Lipschitz exponent reduces with the mass added, but there are definite steps in the exponent. In fact, the reduction in the Lipschitz exponent appears to be less than expected when a rectangular magnet is added to the beam. The magnets add some stiffness to the beam in addition to the mass, and the mass and stiffness have opposite effects on the natural frequencies. However, the change in natural frequency due to added mass, shown in Figure 5 obtained from the experimental response data, shows that the natural frequency changes smoothly with the mass added.
TABLE 1: The properties of the bladed disk.

\begin{tabular}{lc}
\hline$m_{a}$ & $1 \mathrm{~kg}$ \\
$m_{d}$ & $1 \mathrm{~kg}$ \\
$k_{a}$ & $10 \mathrm{kN} / \mathrm{m}$ \\
$k_{d}$ & $20 \mathrm{kN} / \mathrm{m}$ \\
$k_{c}$ & $0.2 k_{a} \mathrm{kN} / \mathrm{m}$ \\
$n_{b}$ & 20 \\
\hline
\end{tabular}

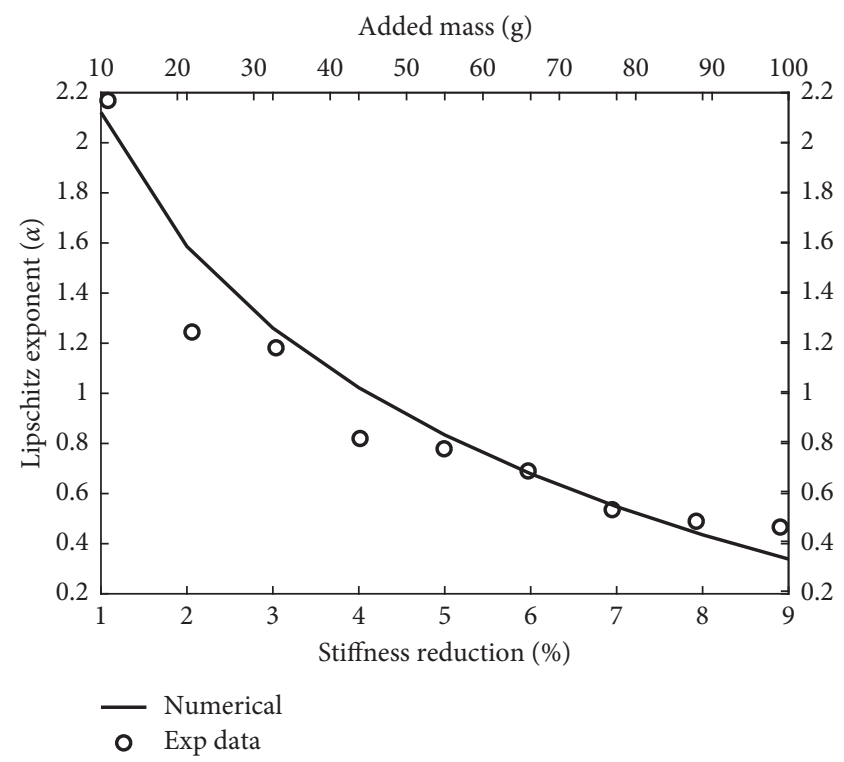

Figure 4: The change in the Lipschitz exponent due to damage severity.

TABLE 2: The position of the masses added to the blade for the different damage cases in the bladed disk experiment.

\begin{tabular}{lcc}
\hline Mass added & Rectangular magnets $(33 \mathrm{~g})$ & Round magnets $(11 \mathrm{~g})$ \\
\hline $11 \mathrm{~g}$ & - & Top \\
$22 \mathrm{~g}$ & - & Top + bottom \\
$33 \mathrm{~g}$ & Top & - \\
$44 \mathrm{~g}$ & Top & Top \\
$55 \mathrm{~g}$ & Top & Top $\times 2$ \\
$66 \mathrm{~g}$ & Top + bottom & - \\
$77 \mathrm{~g}$ & Top + bottom & Top \\
$88 \mathrm{~g}$ & Top + bottom & Top + bottom \\
$99 \mathrm{~g}$ & Top $\times 3$ & - \\
\hline
\end{tabular}

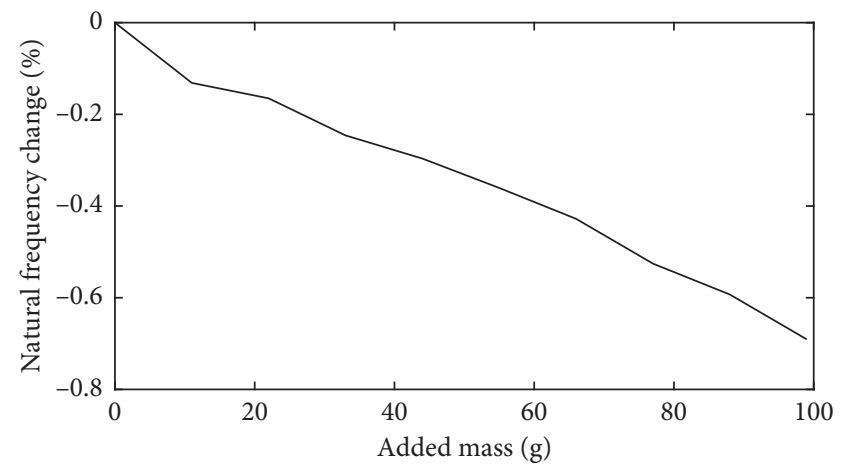

FIgURE 5: The change in the experimental natural frequency due to mass added. 
3.3. Comparing Simulated and Experimental Results. To compare the simulated and experimental results, a relationship must be found between the stiffness reduction in the simulation and the added mass in the experiment. This relationship was determined by equating the change in the natural frequency due to "damage". In the simulation, the computed change in the first natural frequency for stiffness reduction of $5 \%$ was $0.3267 \%$, whereas the change in the first experimental natural frequency due to an added mass of $55 \mathrm{~g}$ was about $0.36 \%$. Thus, the numerical first natural frequency change due to a $5 \%$ stiffness reduction was close to the experimental first natural frequency change due to the added mass of $55 \mathrm{~g}$. A finite element (FE) analysis of the blade was also performed to give an alternative comparison. Each blade in the disk is considered as a cantilever beam with dimensions $100 \times 31 \times 4 \mathrm{~mm}$. Young's modulus was determined as $166 \mathrm{GPa}$ by model updating to match with the experimental first natural frequency for the undamaged case for a mass density of $7870 \mathrm{~kg} / \mathrm{m}^{3}$. The beam was divided into 20 elements to achieve an accurate estimate of the first natural frequency, and the damage (mass) was introduced from the second to fourth element of the beam to correspond with the location of the added mass in the experiment. The added mass of $55 \mathrm{~g}$ was simulated in the FE model, and the change in the first natural frequency was about $0.3818 \%$ which is approximately equal to the experimental change in the first natural frequency. Thus in Figure 4, a linear relationship between added mass and stiffness reduction is based on the equivalence of a $5 \%$ stiffness reduction and an added mass of $55 \mathrm{~g}$. Figure 4 then shows a reasonable agreement between the experimental results and the numerical results.

\section{Experimental Validation for a Rotating Bladed Disk}

4.1. The Bladed Disk Test Rig. A test rig was designed and manufactured at Swansea University in order to generate BTT measurements using different types of sensors and validate the proposed technique. The sensors were selected to be typical of those used to obtain BTT measurements, i.e., an active eddy current sensor (AECS), a passive eddy current sensor (PECS), and an optical sensor. The test rig is composed of a bladed disk with 12 blades surrounded by a cylindrical casing at the distance $\delta$ (the gap between the blade tip and the sensor). The bladed disk is clamped to the end of a rotating shaft supported by two ball bearings fitted in two split plummer block housings. The shaft is driven by a servo motor at $100 \mathrm{rpm}$. The manufactured test rig details are shown in Figure 6.

The "damage" is induced by adding mass to one of the blades. The sensors were mounted to the casing to detect the blade tip displacement as it passes in front of the probes, as shown in Figure 7(a). The sensor outputs were measured by a 4-channel Data Physics DAQ system for a period of $3.2 \mathrm{~s}$ with a sampling rate of $10240 \mathrm{~Hz}$. The damage was simulated by the added mass realised as magnets are placed $15 \mathrm{~mm}$ from the root of the blade, as shown in Figure 7(b). In this study, the blades were run at

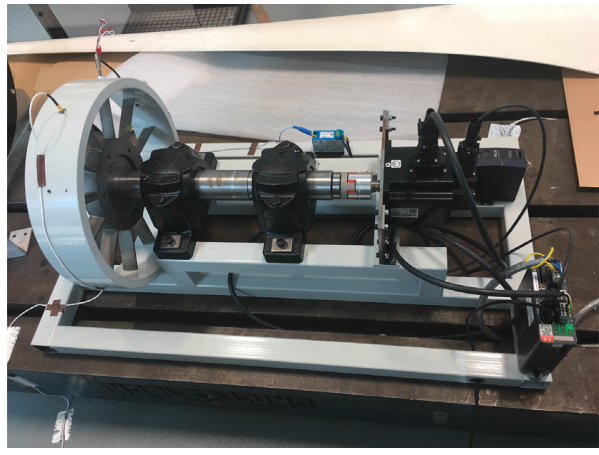

Figure 6: The manufactured test rig.

$100 \mathrm{rpm}$. There was no explicit excitation of the blades in the experiments, although the resolution of the encoder used for the speed control means that the bending vibration of the blades will be excited via the torsional vibration of the shaft. This may be demonstrated by estimating the speed of the machine based on the time of arrival of a particular blade; a nonconstant speed will arise from a combination of the shaft speed variation and the blade vibration. Figure 8 shows a typical example of estimated speed and shows a small variation of $0.03 \%$, which provides sufficient excitation for the WEBMI method. Different test cases were performed where the mass added was changed, i.e., (i) a single added mass to blade 8, (ii) multiple masses added to blades 4 and 8 , and (iii) a single mass added to blade 8 that varied from $11 \mathrm{~g}$ to $99 \mathrm{~g}$; the description of the added masses and their position for the different damage cases is given in Table 2 .

4.2. Signal Processing of Measurements from the BTT Sensors. The raw signal was obtained from the AECS for a speed of $100 \mathrm{rpm}$, as shown in Figure 9 for illustration purposes. The raw signal cannot be directly used in the WPT methodology since the response from individual blades needs to be extracted. The WPT methodology may then be applied to each blade response. One of the major advantages of the proposed approach is that a single noncontact sensor mounted on the stator is sufficient to predict the damage location and severity for all of the blades. The steps to extract individual blade response are as follows:

(i) Initially, the measurements are obtained for $3.2 \mathrm{~s}$, as shown in Figure 9.

(ii) To identify the reference blade, a trial was conducted by removing a blade from the bladed disk assembly. Then the signal was obtained with 11 peaks, and in this case, it is clear which signals correspond to the reference blade and the damaged (added mass) blade from the removed blade signal.

(iii) The reference blade (blade 1) is defined as the blade with the highest amplitude peak, and subsequent peaks were ordered sequentially as the response from the other blades (up to blade 12), for a complete revolution. For illustration, four complete revolutions are considered here and the remaining 


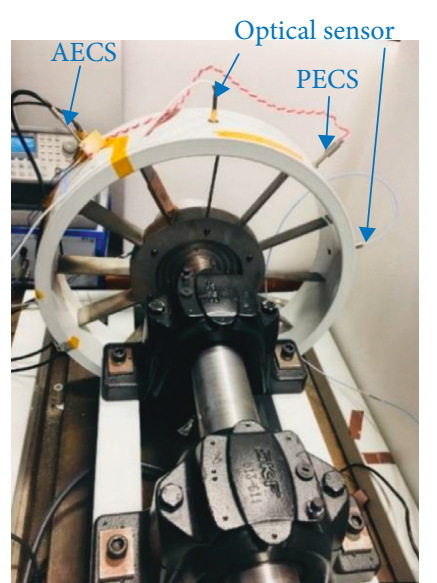

(a)

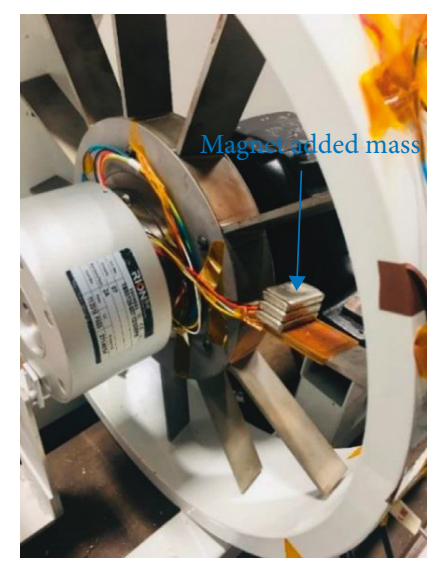

(b)

Figure 7: (a) BTT experimental test rig; (b) blade with magnet added mass.

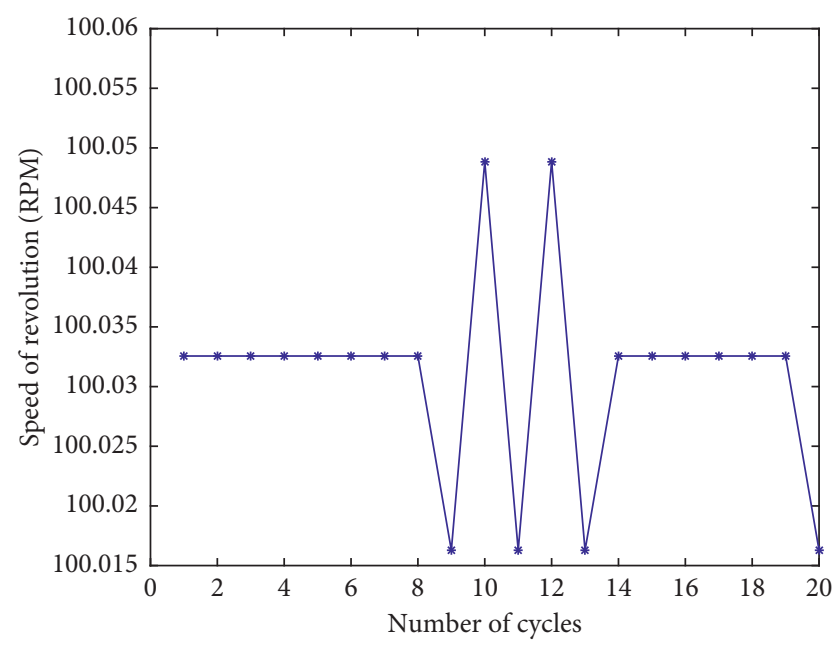

Figure 8: Speed variation.

samples were discarded. More revolutions can be used if required.

(iv) Individual blade responses can be extracted by choosing a fixed sample time that fully contains a single blade response (both before and after the peak) but is less that $T / N$, where $T$ represents one period of rotation and $N$ is the number of blades.

(v) Given knowledge of the number of blades, the responses from all of the blades can be allocated to individual blades and merged over multiple revolutions, as shown in Figure 10 for blade 1.

After processing the signal, each blade BTT signal may be applied into the WPT methodology to detect the damage in the rotating blades. Brief details about the WPT methodology and the formulation of the wavelet energy based mistuning index (WEBMI) were discussed in Section 2.

4.3. Single Added Mass Locations. The sampling frequency of blade response measures from the standard processing of

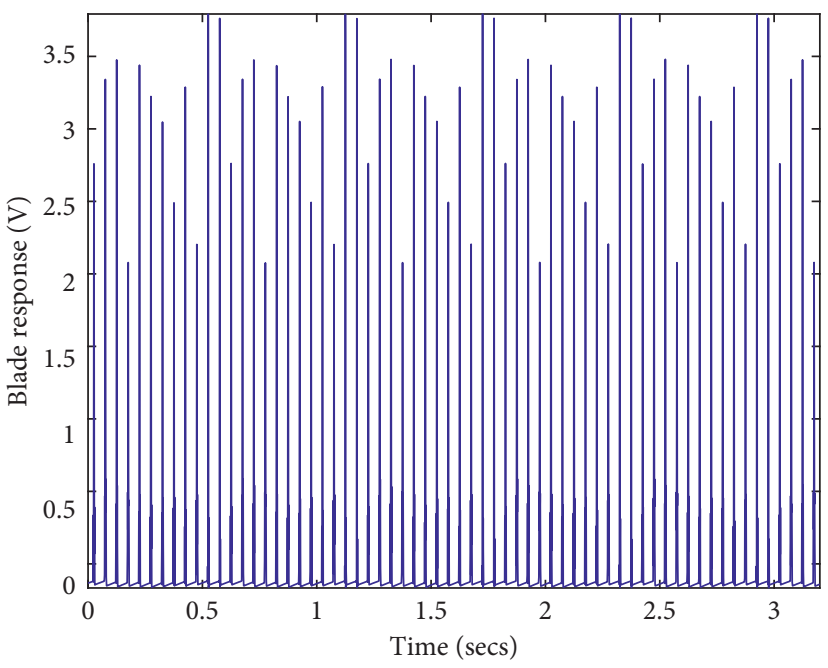

Figure 9: A raw BTT signal from the AECS at a speed of $100 \mathrm{rpm}$.

BTT signals completely depends on the rotating speed of the machine since there is only one measurement for each blade passing. However, this study has considered the sensor output directly at a constant sample rate of $10240 \mathrm{~Hz}$. The damage identification study was conducted for a speed of $100 \mathrm{rpm}$, and the added mass of $99 \mathrm{~g}$ was located at a position of $15 \mathrm{~mm}$ from the root of blade 8 . The signals from the BTT sensors of all blades for the undamaged and damaged cases from the AECS are shown in Figure 11. The sample time for each blade was based on 500 samples (250 each side of the peak), and the reconstructed signals for blade 8 for the undamaged and the damaged cases are shown in Figure 12. Comparing the damaged signal with the undamaged signal, there are small shifts observed in the responses of blade 8 due to the added mass at blade 8 . However, the interpretation of the shift in the signal alone cannot provide a robust damage feature for the rotating blades. Therefore, the signal for each blade was subjected to the WPT method which decomposed the signal into different frequency bandwidth signals to detect the damage effectively. In this study, the wavelet 


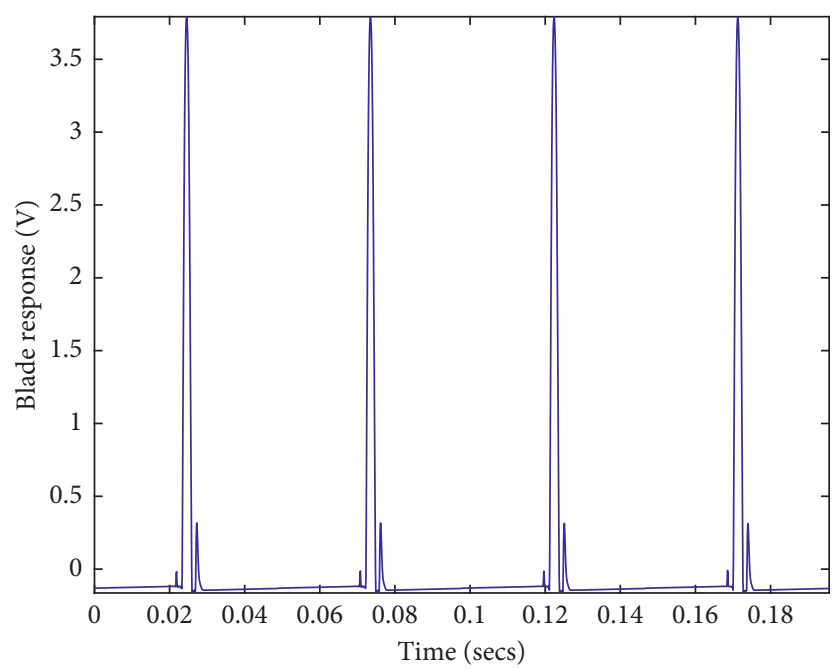

FIGURE 10: Reconstructed blade 1 BTT signal up to four completed revolutions.

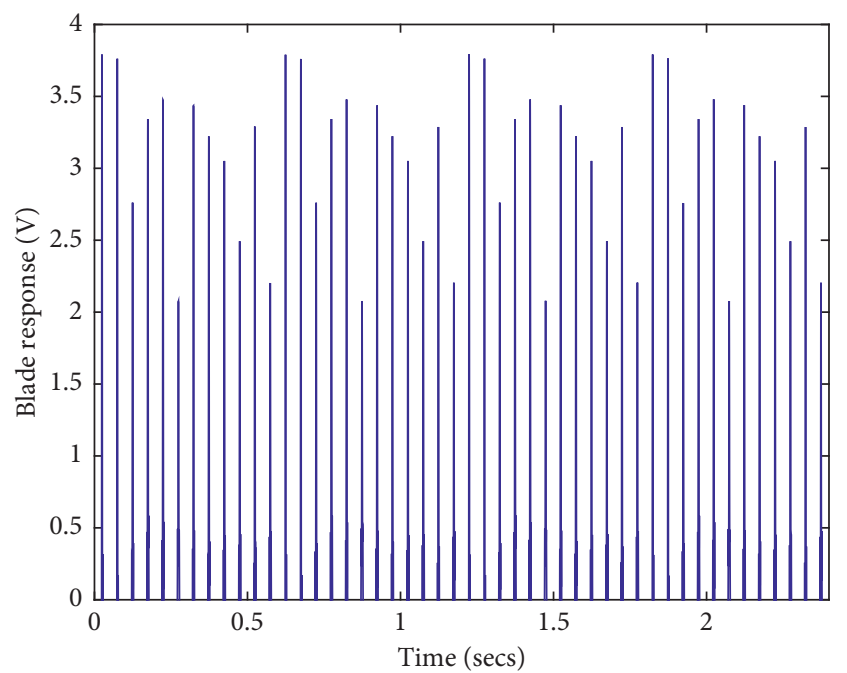

(a)

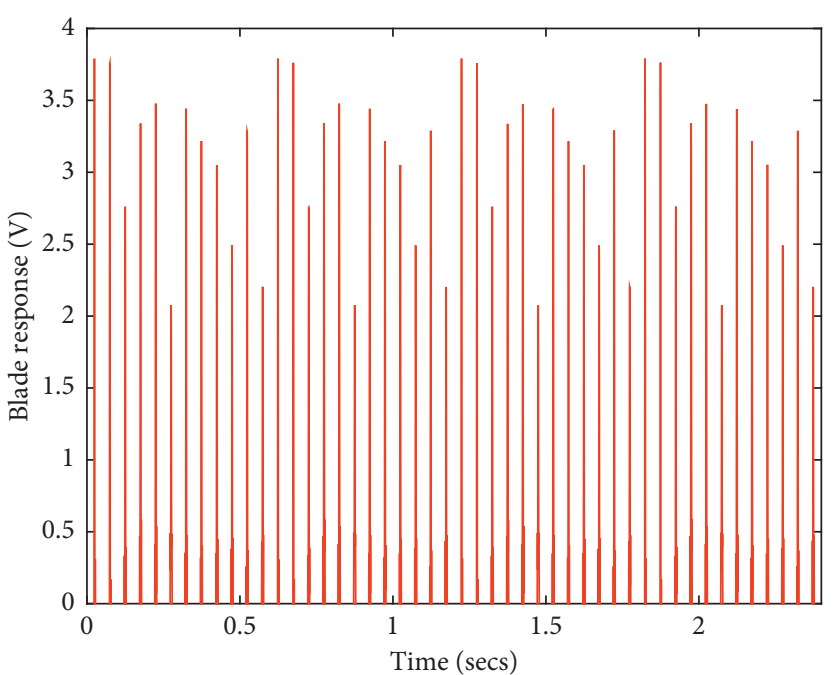

(b)

FIGURE 11: AECS reconstructed BTT signals of all blades for a speed of $100 \mathrm{rpm}$. (a) Undamaged and (b) damaged cases.

function " $d b 6$ " with three vanishing moments was chosen, which satisfied the vanishing moments condition for estimating the damage location and severity in the rotating blades.

In order to select the optimal decomposition level, the Shannon entropy method was employed, as briefly discussed in [19]. Based on this method, the entropy of both approximate and detailed coefficients was estimated at each level. At level 2, the entropy was 0.00933 , which was less than the entropy of the detailed coefficients, which was 0.03069; hence, the decomposition level 2 was selected as an optimal level to detect the changes of $99 \mathrm{~g}$ added mass in the rotating blade. For the case of BTT signals, the vicinity of minute changes can be observed from the approximate component energy, as shown in Figure 13(a). However, it is difficult to predict the damage at the exact location. Thus, the threshold limit with a high confidence interval was applied to the WEBMI, and the resulting WEBMI-UL indicates a substantial peak at the exact location of blade 8 , as shown in Figure 13(b).

In order to check the effectiveness of the other sensors, the PECS and optical sensor were employed simultaneously, in addition to the AECS to monitor the blade tip displacement, as shown in Figure 7(a). The reconstructed BTT signals of the undamaged and the damaged blade 8 from the PECS and the optical sensors are shown in Figures 14 and 15 . There is hardly any change found in the undamaged BTT signal of blade 8 (see Figure 14(a)) from the PECS, whereas for the damaged signal of blade 8 , Figure 14(b) shows subtle changes in the time response due to the added mass placed on blade 8 . In fact, the amplitudes of the blade response measured from the PECS are very low compared to the AECS and the optical sensor. However, this will not affect the damage identification process. On the other hand, the optical sensor BTT response of undamaged blade 8 (see Figure 15(a)) shows no changes, but 


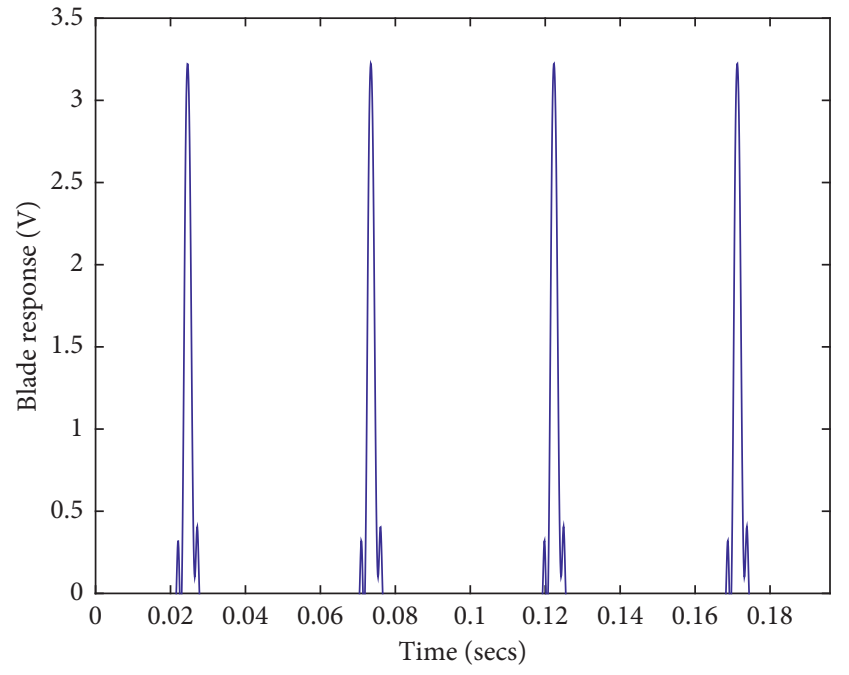

(a)

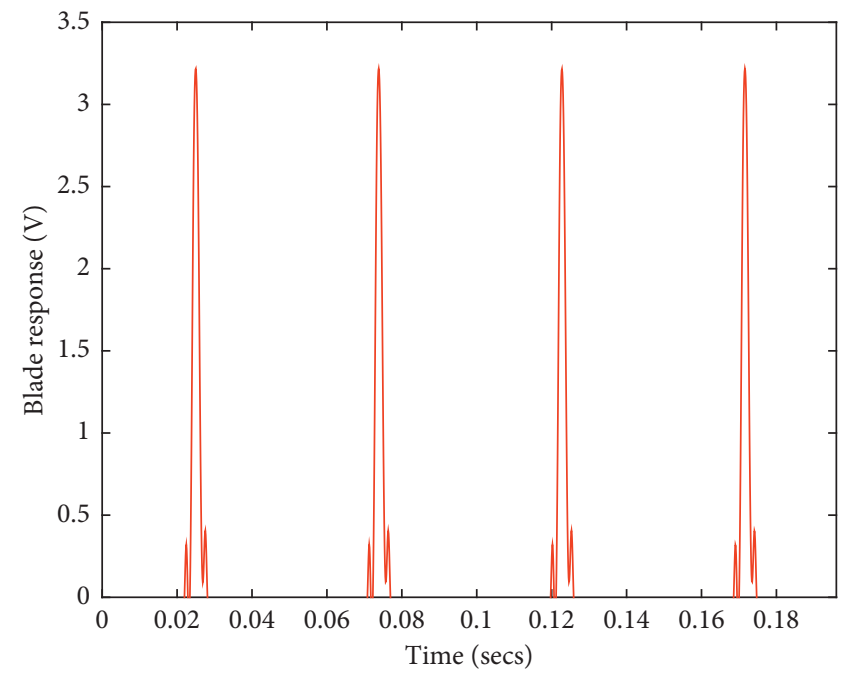

(b)

FIgURE 12: AECS reconstructed BTT signals of blade 8 for a speed of $100 \mathrm{rpm}$. (a) Undamaged and (b) damaged cases.

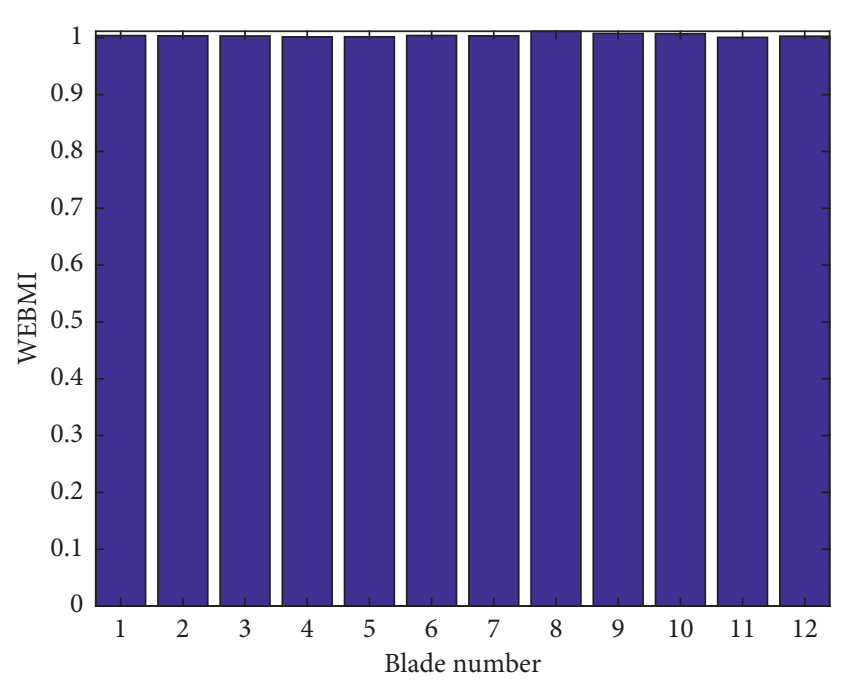

(a)

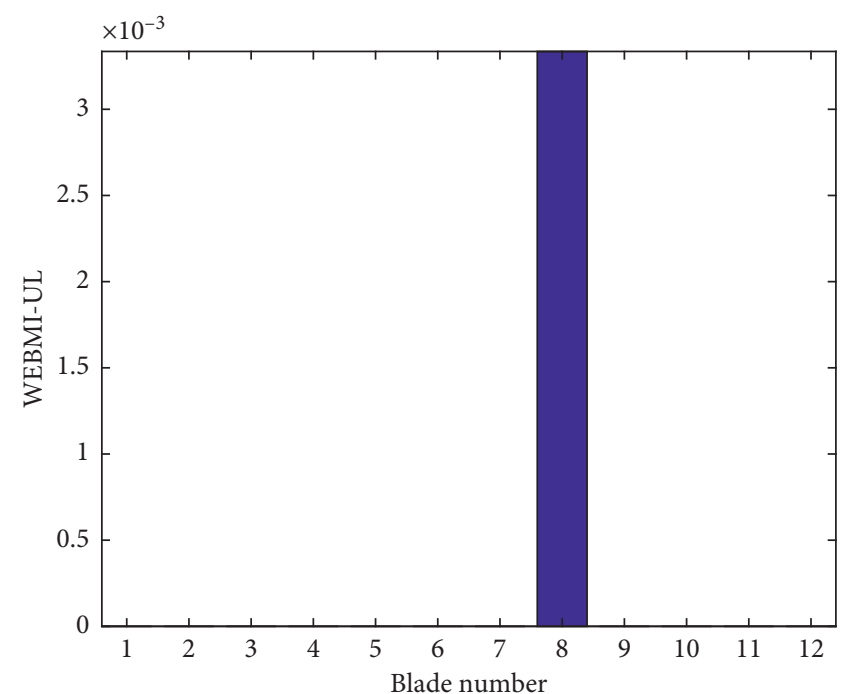

(b)

FIgURE 13: AECS results for the added mass located at blade 8. (a) WEBMI and (b) WEBMI-UL.

the blade 8 damaged response reveals bump characteristics at the root of the BTT signal as shown in Figure 15(b). This phenomenon occurs due to the optical sensor picking up the signal directly from the presence of the magnet. It is observed that the damaged blade BTT signal from the optical sensor provides the trend for the damage existence and location. Still, a robust damage identification methodology is required to predict the exact damage location. Thus, the BTT signals were applied to the WPT methodology which required a level 3 decomposition to detect the exact damage (added mass) location in the rotating blades. It is found that the estimated WEBMI is not distinct for the damaged blade corresponding to the PECS and the optical sensor cases, as shown in Figures 16(a) and 17(a). Therefore, the statistical threshold limit was applied to the WEBMI to pinpoint the exact location of the damage, as shown in Figures 16(b) and $17(\mathrm{~b})$.

4.4. Multiple Added Masses Locations. The WPT methodology has identified the single added mass location in the rotating blades at constant speed and different sensors cases. In practice, damage may occur in more than one location in the rotating bladed disk. Hence, this study extends the WPT identification methodology for the case of multiple damaged blades. The BTT measurements were performed for two added masses (each $66 \mathrm{~g}$ ) located near the roots of blade 4 and blade 8 . This study was conducted at $100 \mathrm{rpm}$. The reconstructed BTT signals were obtained for blades 4 and 8 from the three sensors and are shown in Figures 18(a)-18(f). 


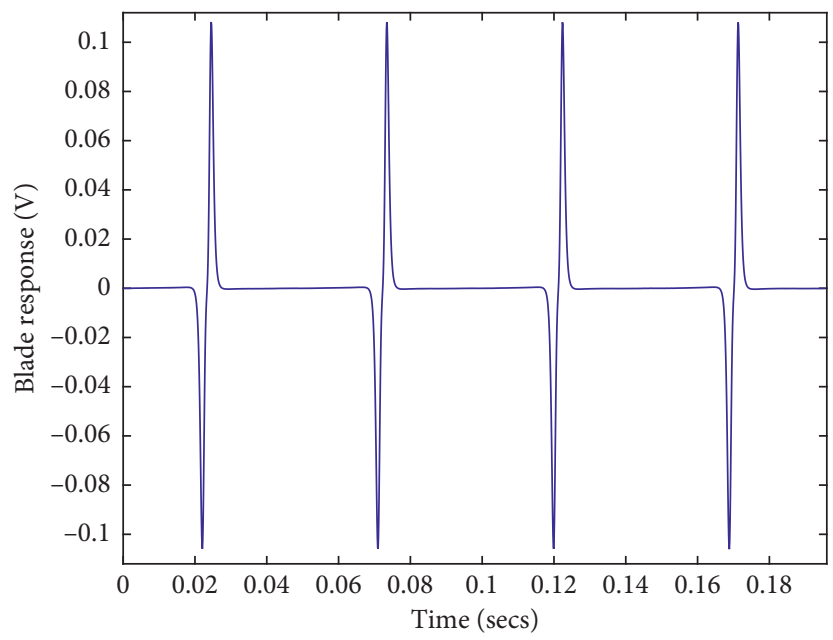

(a)

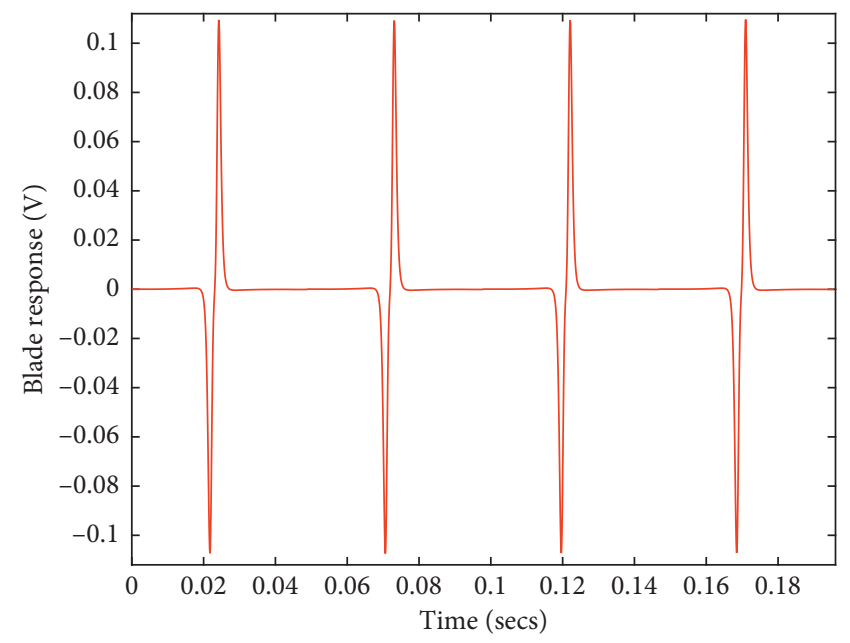

(b)

Figure 14: PECS reconstructed BTT signals of blade 8 for a speed of $100 \mathrm{rpm}$. (a) Undamaged and (b) damaged cases.

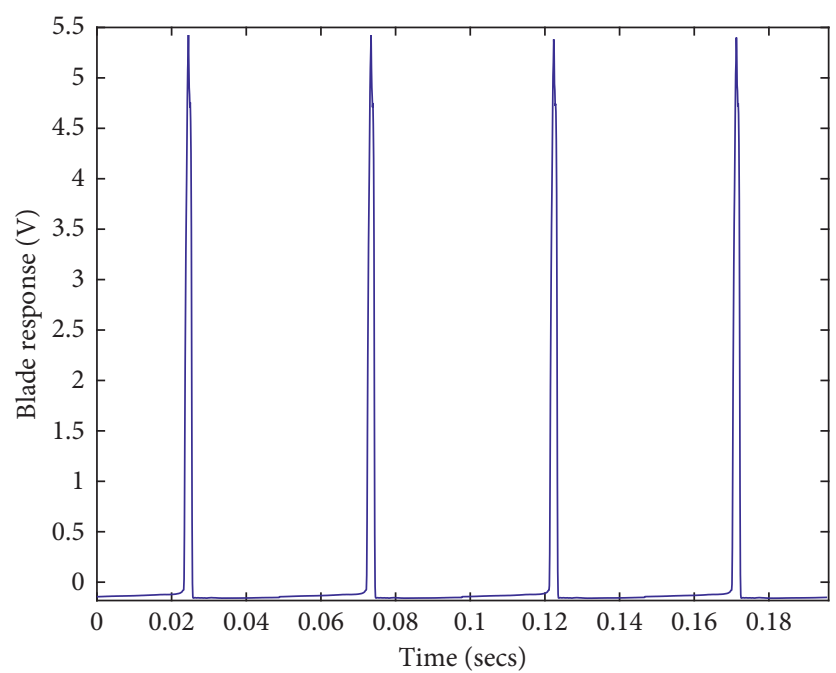

(a)

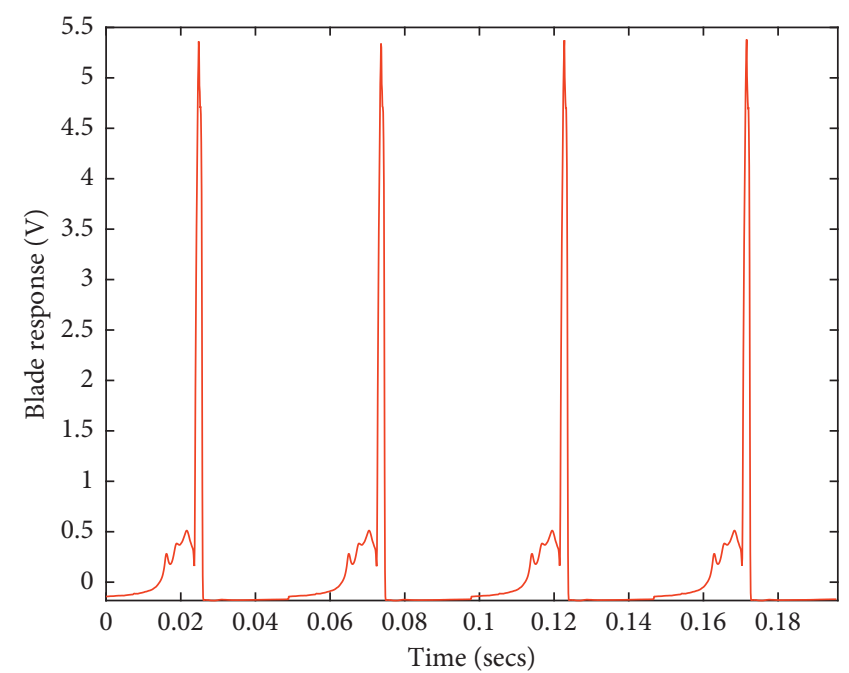

(b)

FIGURE 15: Optical sensor reconstructed BTT signals of blade 8 for a speed of $100 \mathrm{rpm}$. (a) Undamaged and (b) damaged cases.

There are some apparent shifts found in the BTT signals as shown in Figures 18(a), 18(d), and 18(f), whereas only subtle shifts are observed in Figures 18(b), 18(c), and 18(e). However, it is challenging to detect damage from the shift in the BTT responses. Therefore, the BTT signals were subjected to the WPT analysis with the choice of the " $d b 6$ " wavelet function which required a level 6 decomposition. It is challenging to detect the damage (added masses) at multiple locations using the AECS and PECS results even after trying with the different component energies. For the last component energy, the peaks are distinct only at single location of the added mass, as shown in Figures 19 and 20. Yet, the optical sensor can detect the damage (added masses) at multiple locations at blades 4 and 8, as shown in Figure 21. Therefore, the optical sensor is more reliable than the AECS and the PECS sensors to reveal the subtle changes in the multiple locations from the BTT signals.
4.5. Estimation of Damage Severity. Damage severity is the third level of the damage identification methodology after the identification of damage existence and the location of damage. The Lipschitz exponent " $\alpha$ " was derived from the WPT analysis to estimate the damage severity in the rotating blade. The formulation of the Lipschitz exponent " $\alpha$ " is briefly explained in Sections 2.3 and 2.4. This section discusses the quantification of damage (added mass) severity in the rotating blade based on the BTT measurements. Therefore, the BTT experiments were performed with the added mass varied from $11 \mathrm{~g}$ to $99 \mathrm{~g}$ with an increment of $11 \mathrm{~g}$ at blade 8 for a constant speed of $100 \mathrm{rpm}$. As we observed from Section 4.4, the optical sensor is robust to predict the minute changes in the BTT signals. Hence, the following discussion regarding the damage severity is based on the optical sensor results.

In this study, the wavelet function " $d b 6$ " was chosen which has three vanishing moments, and the first wavelet 


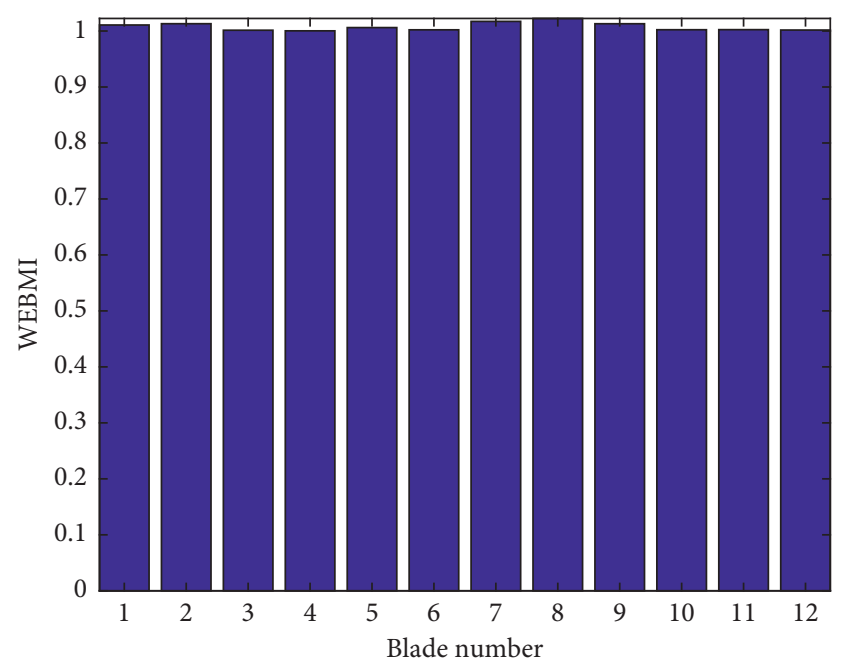

(a)

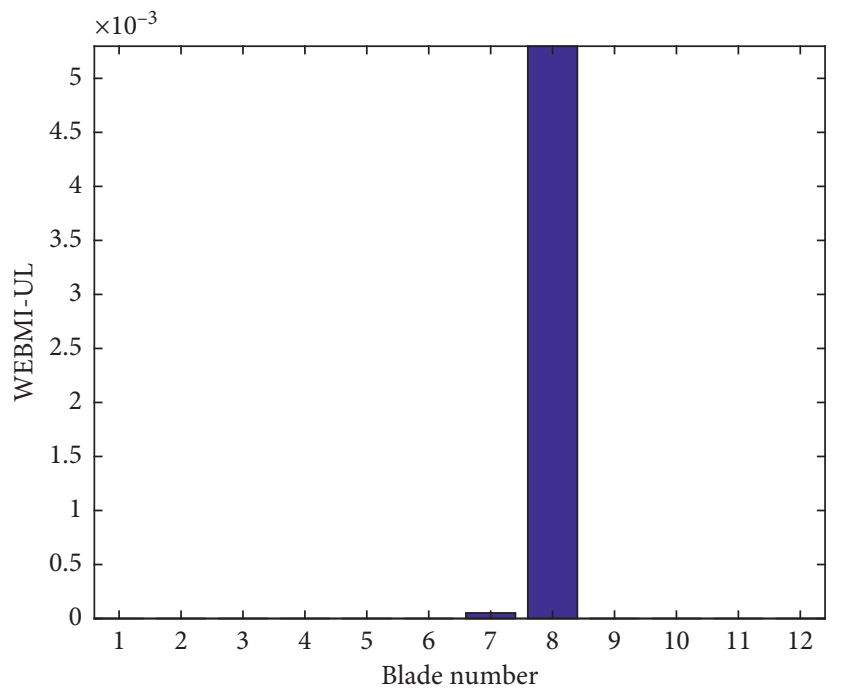

(b)

FIgUre 16: PECS results for the added mass located at blade 8. (a) WEBMI and (b) WEBMI-UL.

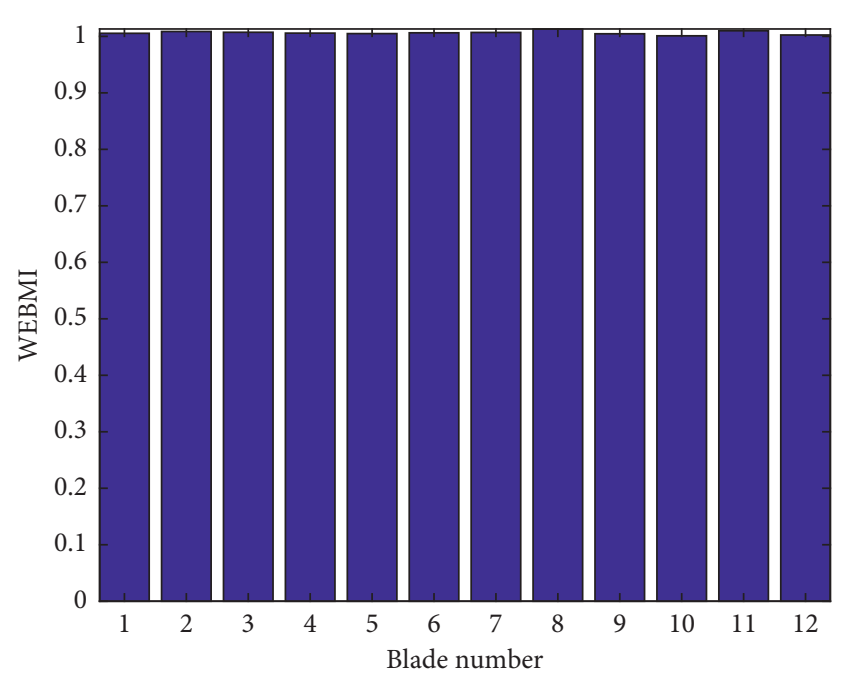

(a)

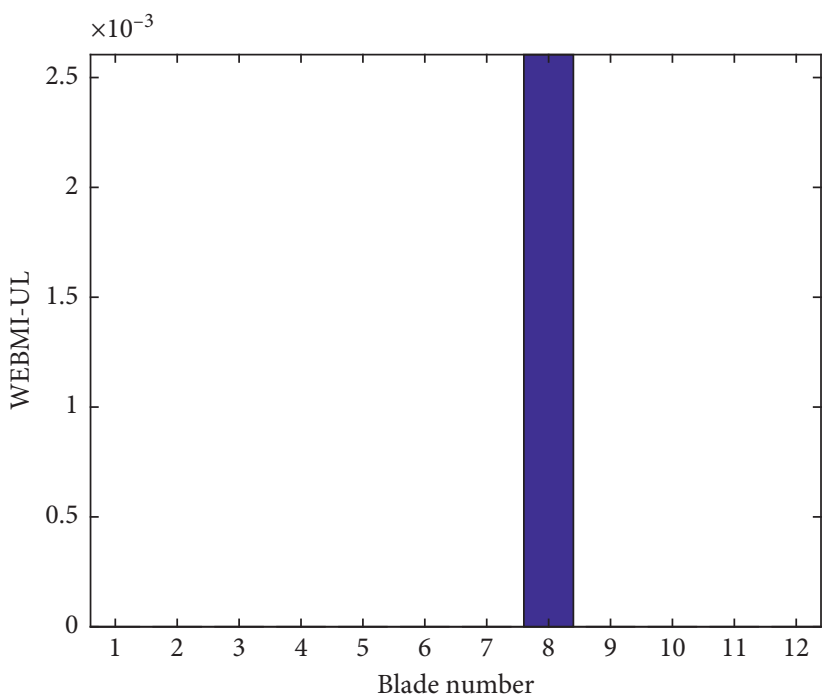

(b)

FIgURE 17: Optical sensor results for the added mass located at blade 8. (a) WEBMI and (b) WEBMI-UL.

packet energy was considered in the damage identification methodology as shown in Figure 1. Then, the Lipschitz exponent " $\alpha$ " was calculated with the input of two different levels (e.g., $j=5$ and $j=9$ ) of coefficients using the relation given in Section 2.4. It is observed that the Lipschitz exponent " $\alpha$ " decreases monotonically as the added mass increases, which means the differentiability of the function decreases as the damage severity increases, as shown in Figure 22. An approximately uniform drop is found up to an added mass of $55 \mathrm{~g}$ which indicates the sensitivity of the Lipschitz exponent due to the change in mass up to $55 \mathrm{~g}$. However, this behavior decays slowly as added mass increases. In addition, Figure 22 shows that the experimental Lipschitz exponent approximately follows the behavior of a cubic polynomial with an $R^{2}$ value of 0.9985 . The Lipschitz exponent “ $\alpha$ ” has an ability to predict the differentiability of the function for an added mass as small as $11 \mathrm{~g}$. The Lipschitz exponent $\alpha \leq n$ obeys the vanishing moments condition which means the chosen " $d b 6$ " wavelet function was appropriate for this study. In order to predict less than $11 \mathrm{~g}$ of added mass, even higher-order wavelet functions are desirable. Thus, the Lipschitz exponent is a promising index to quantify the damage severity in rotating blades based on the BTT measurements.

\section{Conclusions}

This study has considered WPT-based damage identification in rotating blades and bladed disk using BTT measurements 


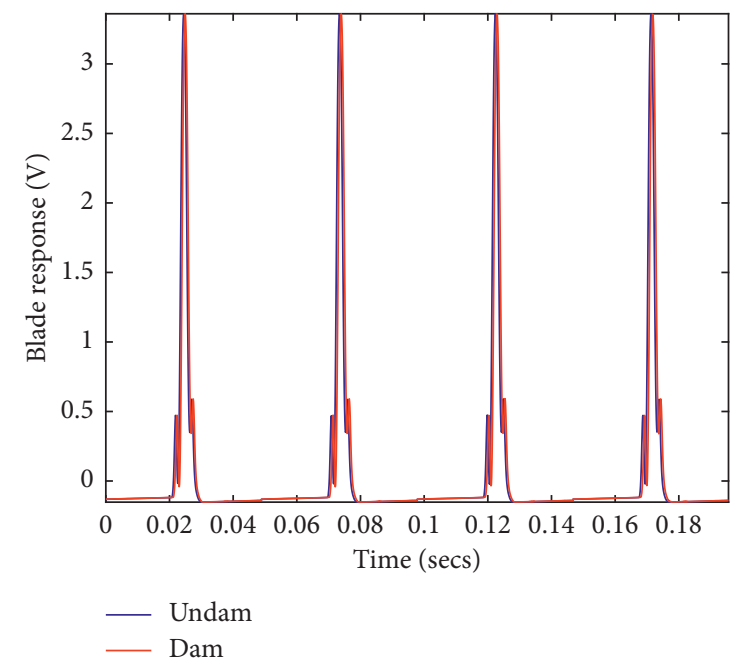

(a)

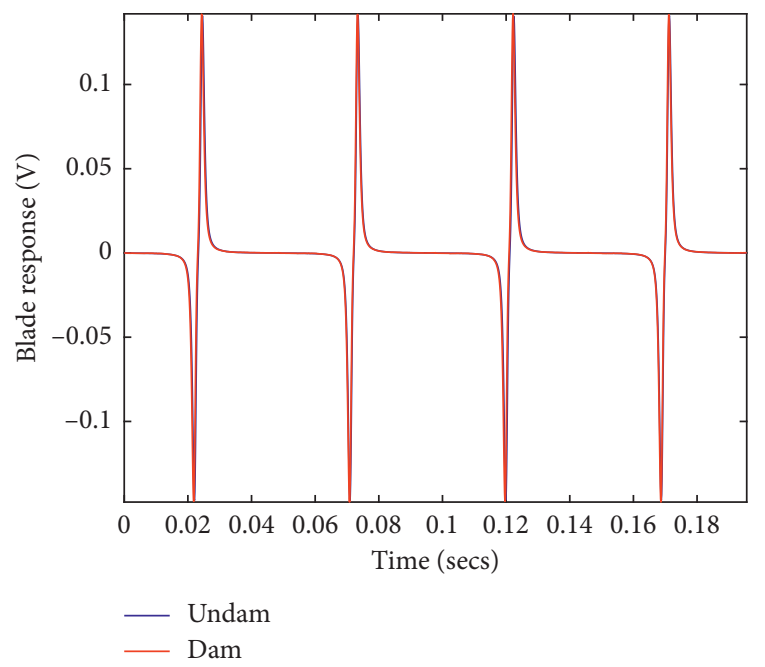

(c)

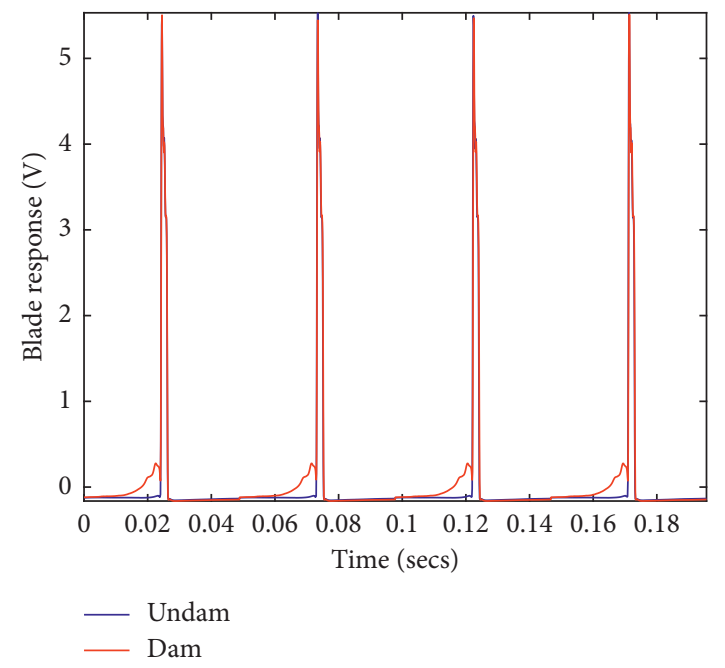

(e)

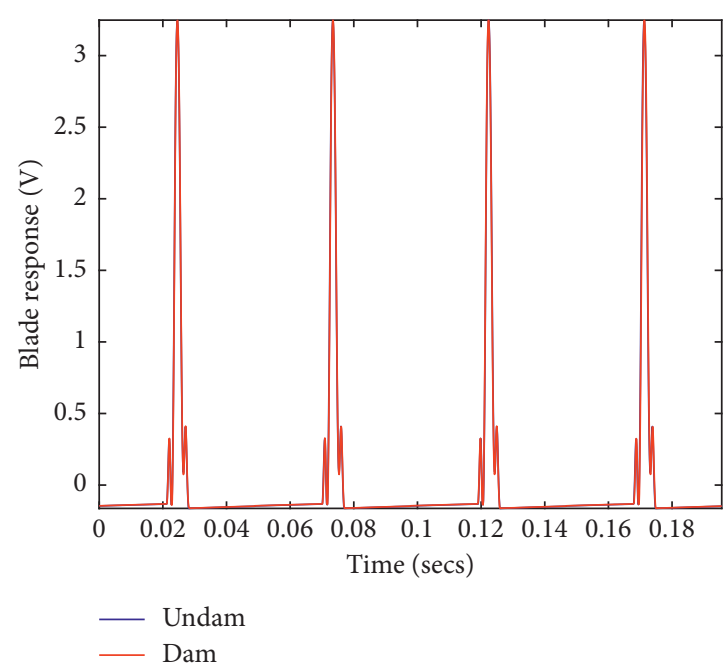

(b)

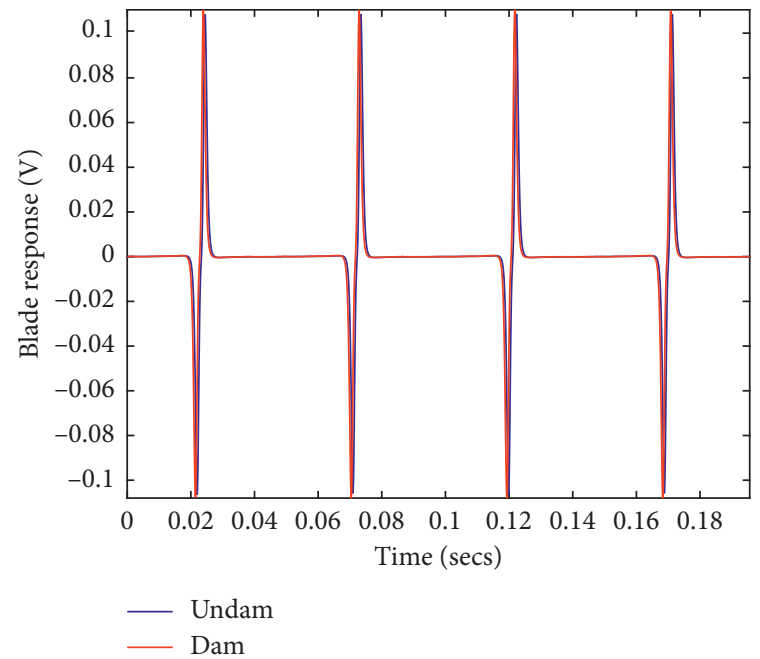

(d)

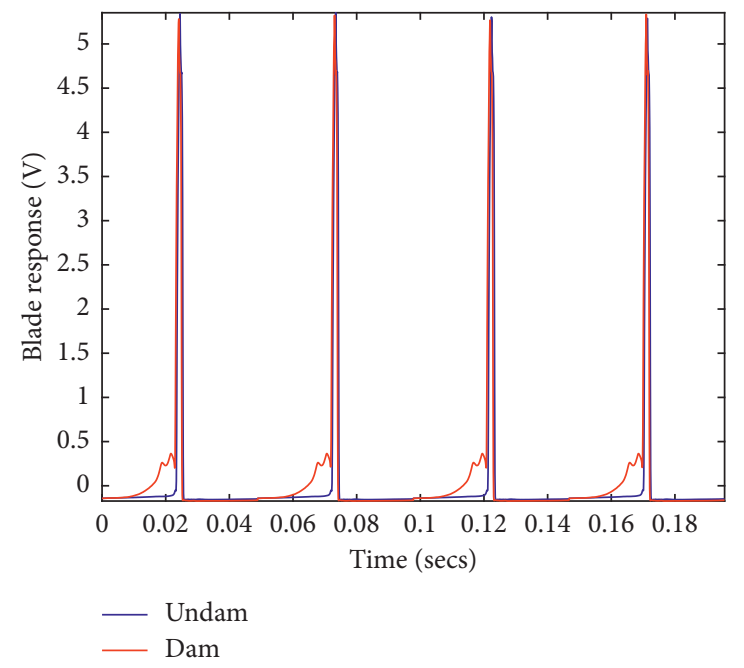

(f)

Figure 18: Reconstructed BTT signals of undamaged and damaged cases of blades 4 and 8 from (a, b) AECS, (c, d) PECS, and (e, f) optical sensor, respectively. 


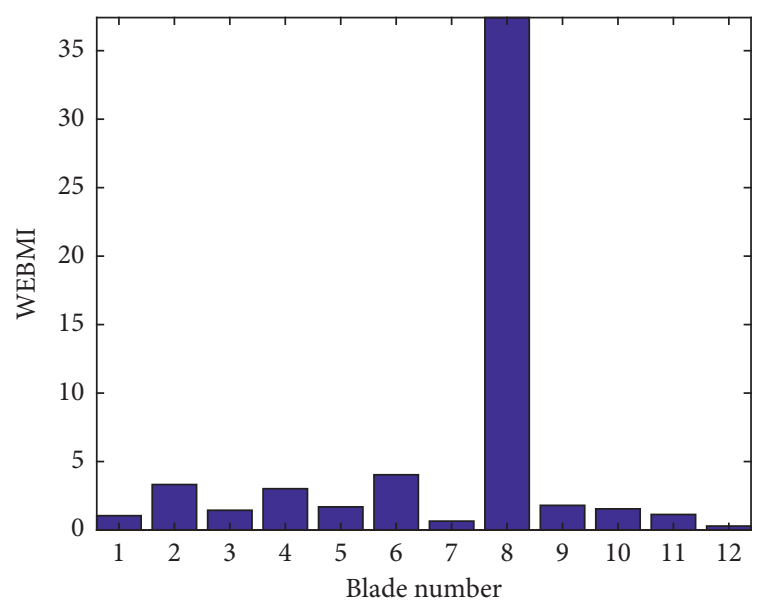

(a)

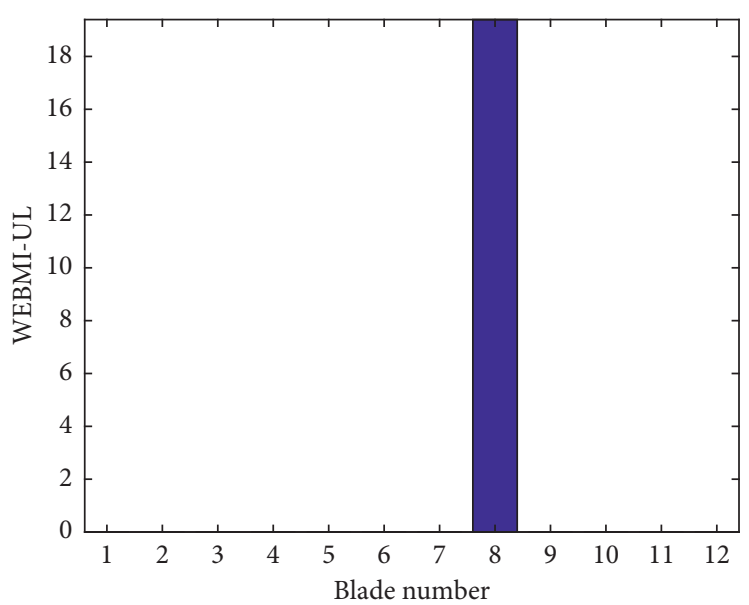

(b)

FIGURE 19: AECS results for the added mass located at blades 4 and 8. (a) WEBMI and (b) WEBMI-UL.

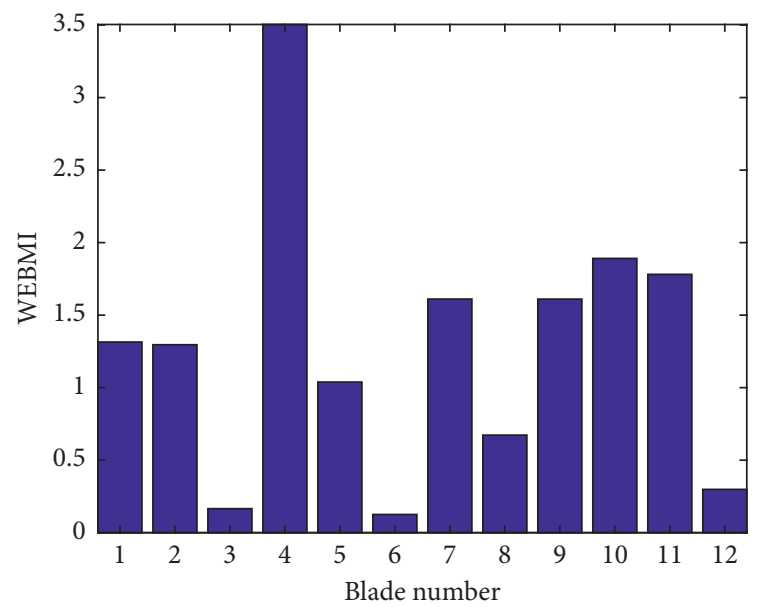

(a)

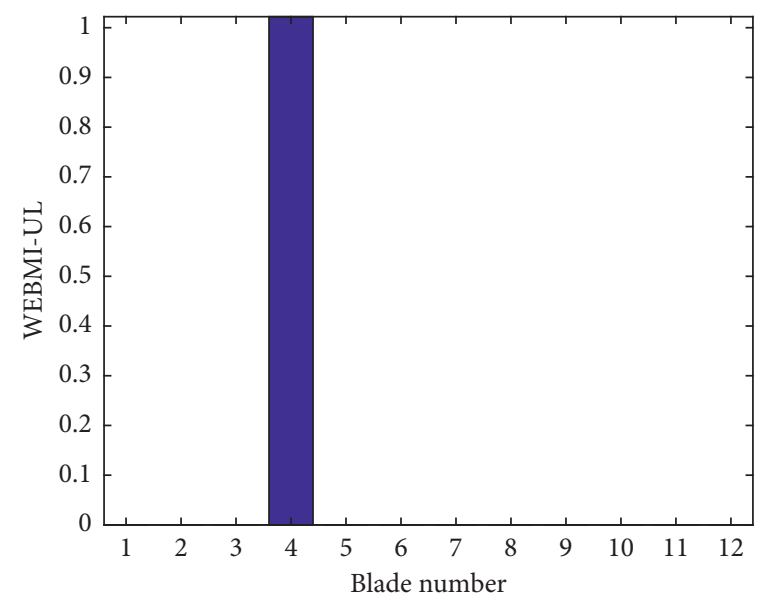

(b)

FIgURE 20: PECS results for the added mass located at blades 4 and 8. (a) WEBMI and (b) WEBMI-UL.

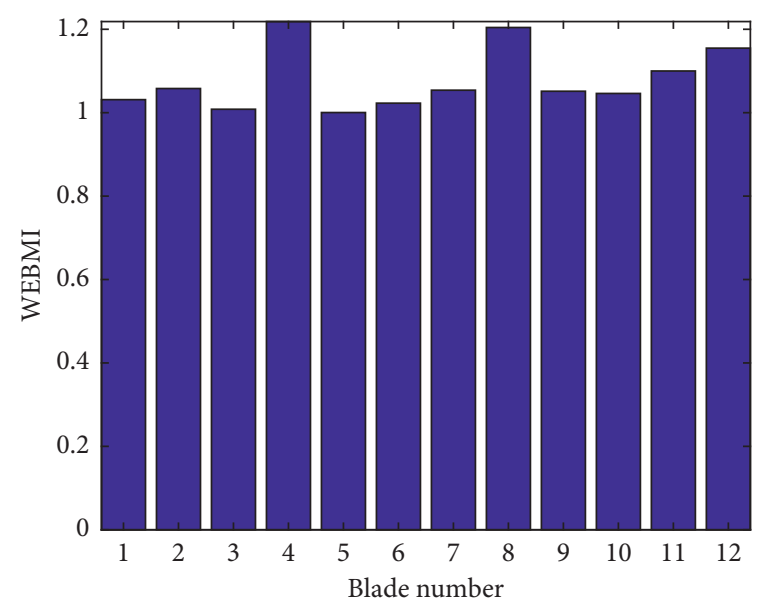

(a)

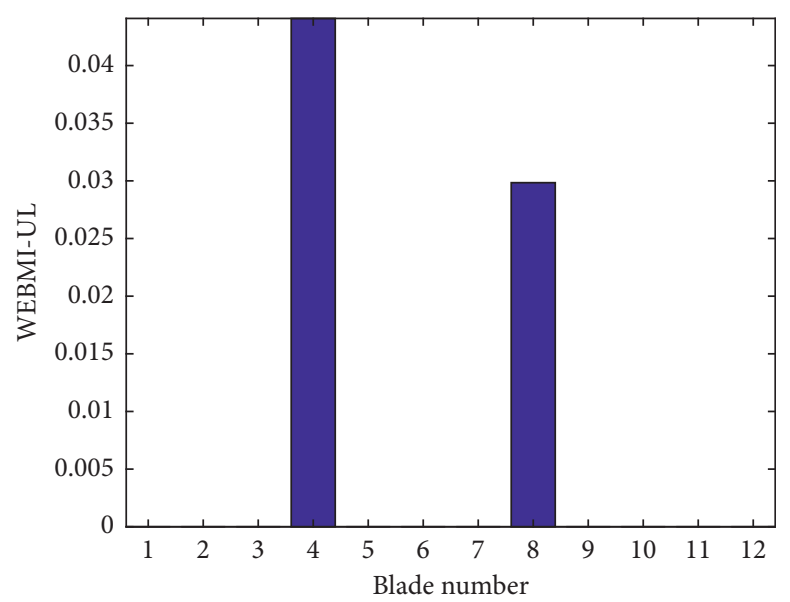

(b)

FIgURE 21: Optical sensor results for the added mass located at blades 4 and 8. (a) WEBMI and (b) WEBMI-UL. 


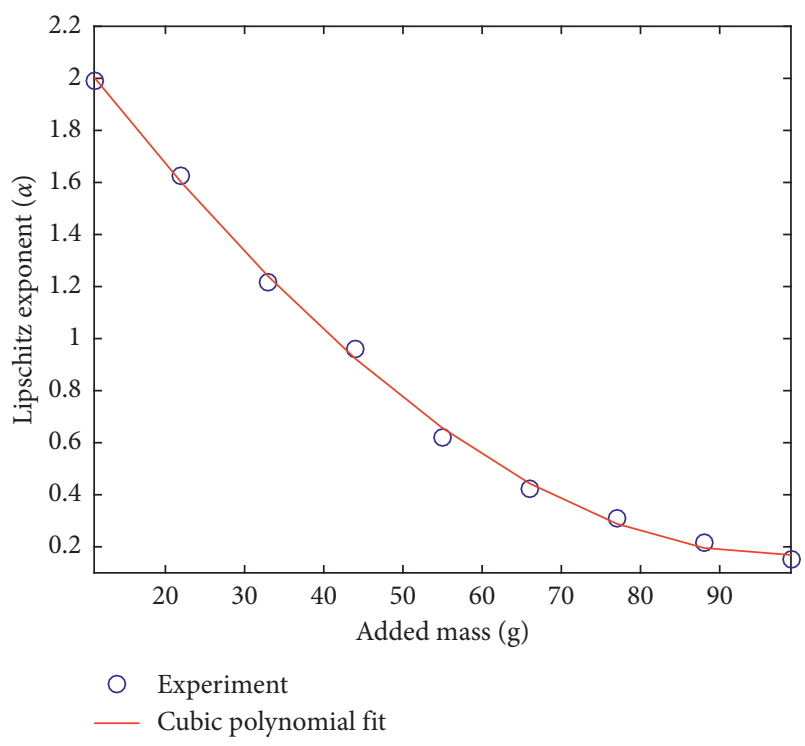

Figure 22: The change in the experimental Lipschitz exponent due to damage severity.

and impact hammer tests. To check the feasibility of the WEBMI for the rotating blades, the BTT experiments were conducted at $100 \mathrm{rpm}$ using three sensors, for different levels of added mass and locations. The signal processing of the raw BTT signal was performed before applying the WPT analysis. The reconstructed BTT signal of each blade was then applied to the WPT methodology to estimate the WEBMI. It is observed that the WEBMI can identify the damage (added mass) presence and location in the rotating blades. The optical sensor is robust to predict the multiple added masses locations and the small damage severity cases, compared to the AECS and the PECS. The Lipschitz exponent " $\alpha$ " was employed to estimate the damage severity in both static bladed disk and the rotating blades. Numerical simulations were performed on a nonrotating bladed disk for various stiffness reductions that ranged from $1 \%$ to $9 \%$. It was found that the experimental results have good agreement with the numerical results. A relationship between the stiffness reduction in the simulations and the added mass in the experiments was determined, and it was concluded that the added mass used in this study is approximately equivalent to the corresponding stiffness reduction in the bladed disk. For the case of rotating blades, the experimental Lipschitz exponent " $\alpha$ " decreases monotonically as the damage severity (added mass) increases and shows good agreement with a cubic polynomial curve fit. Hence, the proposed WEBMI is a promising diagnosis tool to detect the damage presence, location, and the severity in the bladed disk and rotating blades.

\section{Data Availability}

No data were used to support this study.

\section{Conflicts of Interest}

The authors declare that they have no conflicts of interest.

\section{Acknowledgments}

The authors gratefully acknowledge the support of the Qatar National Research Fund through grant number NPRP 71153-2-432.

\section{References}

[1] A. Von, F. Mathieu, and M. P. Tappert, "Health monitoring and prognostics of blades and disks with blade tip sensors," in Proceedings of IEEE Aerospace Conference, pp. 433-440, Big Sky, MT, USA, March 2000.

[2] G. Dimitriadis, I. B. Carrington, J. R. Wright, and J. E. Cooper, "Blade-tip timing measurement of synchronous vibrations of rotating bladed assemblies," Mechanical Systems and Signal Processing, vol. 16, no. 4, pp. 599-622, 2002.

[3] V. Georgiev, M. Holk, V. Kraus et al., "The blade flutter measurement based on the blade tip timing method," in Proceedings of the 15th WSEAS International Conference on System, pp. 270-275, Corfu Island, Greece, July 2011.

[4] S. Madhavan, R. Jain, C. Sujatha, and A. S. Sekhar, "Vibration based damage detection of rotor blades in a gas turbine engine," Engineering Failure Analysis, vol. 46, pp. 26-39, 2014.

[5] G. Battiato, C. M. Firrone, and T. M. Berruti, "Forced response of rotating bladed disks: blade tip-timing measurements," Mechanical Systems and Signal Processing, vol. 85, pp. 912-926, 2017.

[6] K. S. Chana and D. N. Cardwell, "The use of eddy current sensor based blade tip timing for FOD detection," in Proceedings of ASME Controls, Diagnostics and Instrumentation, pp. 169-178, Berlin, Germany, June 2008.

[7] P. Prochzka and F. Vank, "Contactless diagnostics of turbine blade vibration and damage," Journal of Physics: Conference Series, vol. 305, article 012116, 2011.

[8] M. Woike, A. Abdul-Aziz, N. Oza, and B. Matthews, "New sensors and techniques for the structural health monitoring of propulsion systems," Scientific World Journal, vol. 2013, Article ID 596506, 10 pages, 2013.

[9] M. Lackner, Vibration and Crack Detection in Gas Turbine Engine Compressor Blades using Eddy Current Sensors, Massachusetts Institute of Technology, Cambridge, MA, USA, 2002.

[10] S. S. Guru, S. Shylaja, S. Kumar, and R. Murthy, "Pre-emptive rotor blade damage identification by blade tip timing method," Journal of Engineering for Gas Turbines and Power, vol. 136, no. 7, article 72504, 2014.

[11] A. Chatterjee and M. S. Kotambkar, "Modal characteristics of turbine blade packets under lacing wire damage induced mistuning," Journal of Sound and Vibration, vol. 343, pp. 49-70, 2015.

[12] H. Xu, Z. Chen, Y. Yang, L. Tao, and X. Chen, "Effects of crack on vibration characteristics of mistuned rotated blades," Shock and Vibration, vol. 2017, Article ID 1785759, 18 pages, 2017.

[13] B. Salhi, J. Lardis, M. Berthillier, P. Voinis, and C. Bodel, "Modal parameter identification of mistuned bladed disks using tip timing data," Journal of Sound and Vibration, vol. 314, no. 35, pp. 885-906, 2008.

[14] J. Lin, Z. Hu, Z. S. Chen, Y. M. Yang, and H. L. Xu, "Sparse reconstruction of blade tip-timing signals for multi-mode blade vibration monitoring," Mechanical Systems and Signal Processing, vol. 81, pp. 250-258, 2016.

[15] M. Pan, Y. Yang, F. Guan, H. Hu, and H. Xu, "Sparse representation based frequency detection and uncertainty 
reduction in blade tip timing measurement for multi-mode blade vibration monitoring," Sensors, vol. 17, no. 8, pp. 1-19, 2017.

[16] C. Zhongsheng, Y. Yongmin, G. Bin, and H. Zheng, "Blade damage prognosis based on kernel principal component analysis and grey model using subsampled tip-timing signals," Proceedings of the Institution of Mechanical Engineers, Part C: Journal of Mechanical Engineering Science, vol. 228, no. 17, pp. 3178-3185, 2014.

[17] R. Prakash and S. M. Srinivasan, "Rotational mode shape based added mass identification using wavelet packet transform," International Journal for Computational Methods in Engineering Science and Mechanics, vol. 16, no. 3, pp. 182-187, 2015.

[18] P. Rajendran and S. M. Srinivasan, "Identification of added mass in the composite plate structure based on wavelet packet transform," Strain, vol. 52, no. 1, pp. 14-25, 2016.

[19] N. Jamia, P. Rajendran, S. El-Borgi, and M. I. Friswell, "Mistuning identification in a bladed disk using wavelet packet transform," Acta Mechanica, vol. 229, no. 3, pp. 1275-1295, 2018.

[20] L. Montanari, A. Spagnoli, B. Basu, and B. Broderick, "On the effect of spatial sampling in damage detection of cracked beams by continuous wavelet transform," Journal of Sound and Vibration, vol. 345, pp. 233-249, 2015.

[21] J. C. Hong, Y. Y. Kim, H. C. Lee, and Y. W. Lee, "Damage detection using the Lipschitz exponent estimated by the wavelet transform: applications to vibration modes of a beam," International Journal of Solids and Structures, vol. 39, no. 7, pp. 1803-1816, 2002.

[22] E. Douka, S. Loutridis, and A. Trochidis, "Crack identification in beams using wavelet analysis," International Journal of Solids and Structures, vol. 40, no. 13-14, pp. 3557-3569, 2003.

[23] S. Loutridis, E. Douka, and A. Trochidis, "Crack identification in double-cracked beams using wavelet analysis," Journal of Sound and Vibration, vol. 277, no. 13-14, pp. 1025-1039, 2004.

[24] D. M. Reddy and S. Swarnamani, "Damage detection and identification in structures by spatial wavelet based approach," International Journal of Applied Science and Engineering, vol. 10, no. 1, pp. 69-87, 2012.

[25] B. Chen, Y. Kang, P. Li, and W. Xie, "Detection on structural sudden damage using continuous wavelet transform and Lipschitz exponent," Shock and Vibration, vol. 2015, Article ID 832738, 17 pages, 2015.

[26] S. Mallat and S. Zhong, "Characterization of signals from multiscale edges," IEEE Transactions on Pattern Analysis and Machine Intelligence, vol. 14, no. 7, pp. 710-732, 1992.

[27] J. Błaszczuk and Z. Pozorski, "Application of the Lipschitz exponent and the wavelet transform to function discontinuity estimation," Scientific Research of the Institute of Mathematics and Computer Science, vol. 6, no. 1, pp. 23-29, 2007.

[28] A. H. S. Ang and W. H. Tang, Probability Concepts in EngineeringPlanning and Design, Vol. I, John Wiley \& Sons, New York, NY, USA, 1975.

[29] B. Salhi, J. Lardiès, and M. Berthillier, "Identification of modal parameters and aeroelastic coefficients in bladed disk assemblies," Mechanical Systems and Signal Processing, vol. 23, no. 6, pp. 1894-1908, 2009. 


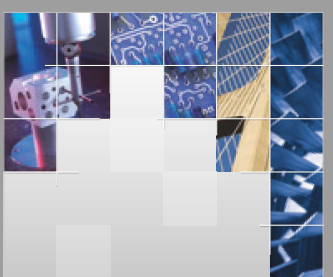

\section{Enfincering}
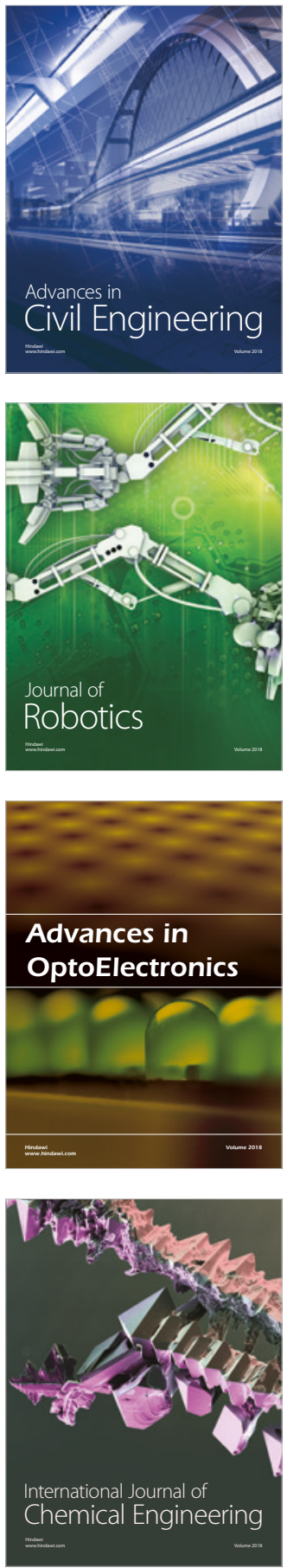

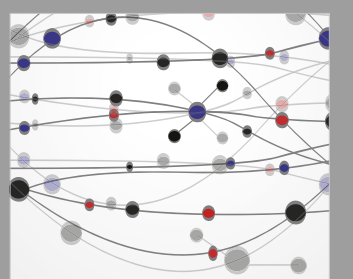

\section{Rotating \\ Machinery}

The Scientific World Journal

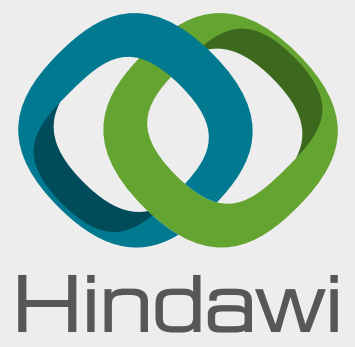

Submit your manuscripts at

www.hindawi.com
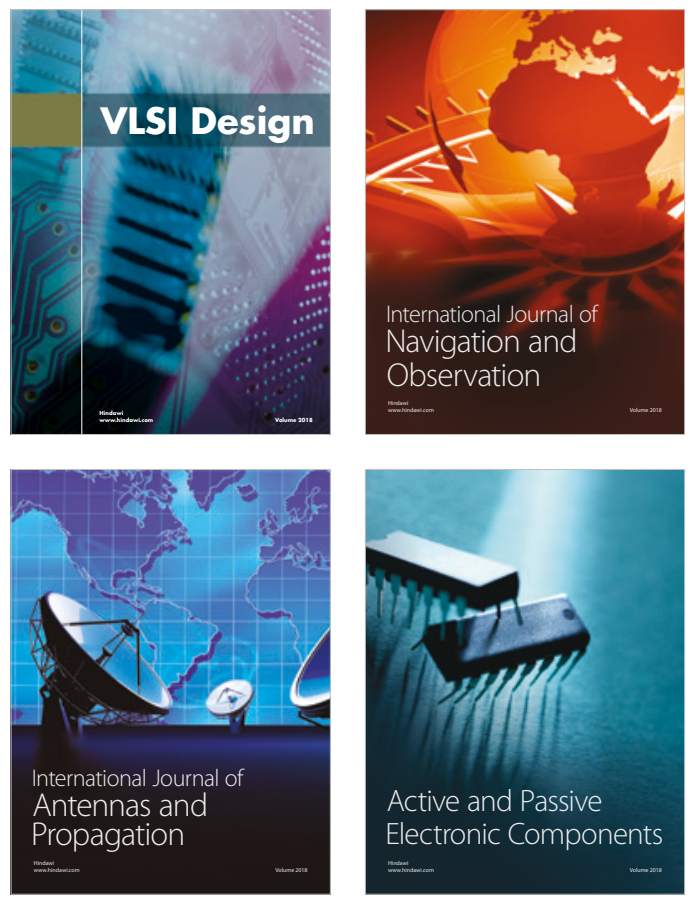
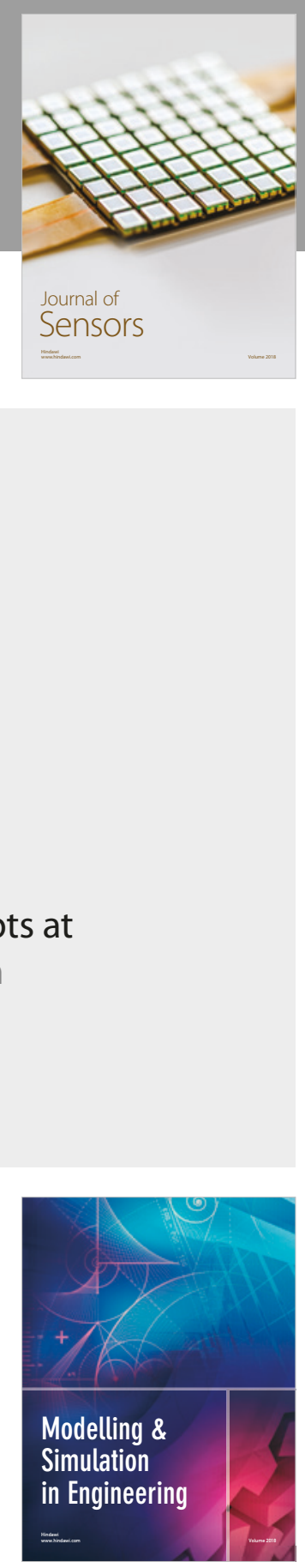

\section{Advances \\ Multimedia}
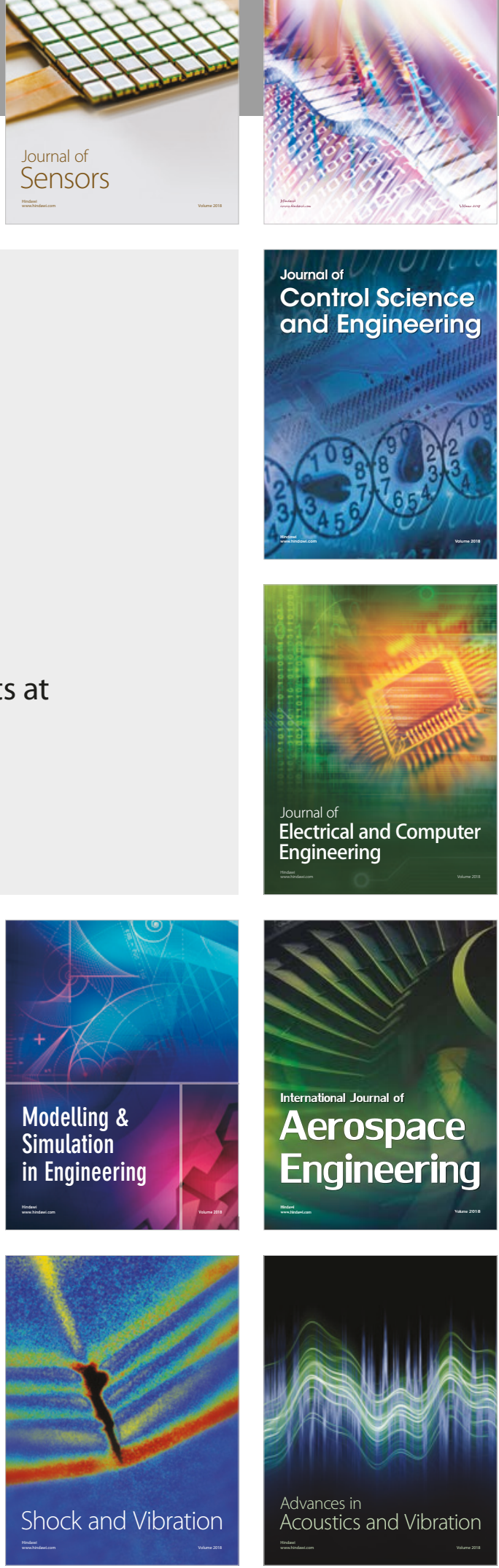